\title{
Cosmological models in scalar tensor theories of gravity and observations: a class of general solutions
}

\author{
M. Demianski ${ }^{1,2}$, E. Piedipalumbo ${ }^{3,4}$, C. Rubano ${ }^{3,4}$, and P. Scudellaro ${ }^{3,4}$ \\ 1 Institute for Theoretical Physics, University of Warsaw, Hoza 69, 00-681 Warsaw, Poland \\ 2 Department of Astronomy, Williams College, Williamstown, MA 01267, USA \\ 3 Dipartimento di Scienze Fisiche, Università di Napoli Federico II, Compl. Univ. Monte S. Angelo, 80126 Naples, Italy \\ e-mail: ester@na.infn.it \\ 4 Istituto Nazionale di Fisica Nucleare, Sez. Napoli, Via Cinthia, Compl. Univ. Monte S. Angelo, 80126 Naples, Italy
}

Received 29 December 2006 / Accepted 6 November 2007

\begin{abstract}
Aims. We study cosmological models in scalar tensor theories of gravity with power-law potentials as models of an accelerating universe.

Methods. We consider cosmological models in scalar tensor theories of gravity that describe an accelerating universe and study a family of inverse power-law potentials, for which exact solutions of the Einstein equations are known. We also compare theoretical predictions of our models with observations. For this we use the following data: the publicly available catalogs of type Ia supernovae and high redshift gamma ray bursts, the parameters of large-scale structure determined by the 2-degree Field Galaxy Redshift Survey (2dFGRS), and measurements of cosmological distances based on the Sunyaev-Zel'dovich effect, among others.

Results. We present a class of cosmological models that describe the evolution of a homogeneous and isotropic universe filled with dust-like matter and a scalar field that is non minimally-coupled to gravity. We show that this class of models depends on three parameters: $V_{0}$ - the amplitude of the scalar field potential, $\widehat{H}_{0}$ - the present value of the Hubble constant, and a real parameter $s$ that determines the overall evolution of the universe. It turns out that these models have a very interesting feature naturally producing an epoch of accelerated expansion. We fix the values of these parameters by comparing predictions of our model with observational data. It turns out that our model is compatible with the presently available observational data.
\end{abstract}

Key words. cosmology: theory - cosmology: cosmological parameters - cosmology: observations

\section{Introduction}

Recent observations of the type Ia supernovae and CMB anisotropy indicate that the total matter-energy density of the universe is now dominated by some kind of dark energy causing an accelerated expansion of the Universe (Perlmutter 1997; Riess et al. 1998, 2004; Spergel et al. 2006). The origin and nature of this dark energy remains unknown (Zeldovich 1967; Weinberg 1989).

Prompted by this discovery, a new class of cosmological models has recently been proposed. In these models the standard cosmological constant $\Lambda$-term is replaced by a dynamical, time-dependent component - quintessence or dark energy - that is added to baryons, cold dark matter (CDM), photons, and neutrinos. The equation of state of the dark energy is assumed to be of a hydrodynamical type $w_{\phi} \equiv \rho_{\phi} / p_{\phi}$, where $\rho_{\phi}$ and $p_{\phi}$ are, respectively, the energy density and pressure, and $-1 \leq w_{\phi}<0$, which implies a negative contribution to the total pressure of the cosmic fluid. When $w_{\phi}=-1$, we recover the standard cosmological constant term. One of the possible physical realization of quintessence is a cosmic scalar field (Caldwell et al. 1998), which induces dynamically a repulsive gravitational force that is responsible for the observed now accelerated expansion of the universe.

The existence of dark energy, which now dominates the overall energy density in the universe, is posing several theoretical problems. First, it is natural to ask why we observe the universe at exactly the time when the dark energy dominates matter (cosmic coincidence problem). The second issue, a fine-tuning problem, arises from the fact that if the dark energy is constant, such as in the standard cosmological constant scenario, then at the beginning of the radiation era its energy density should have been vanishingly small in comparison with the radiation and matter component. This poses a problem, since to explain the inflationary behavior of the early universe and the late time dark energy dominated regime, the dark energy should evolve and cannot simply be a constant. All these circumstances stimulated a renewed interest in the generalized gravity theories, and prompted consideration of a variable $\Lambda$ term in more general classes of theories, such as the scalar tensor theories of gravity.

In our earlier paper (Demianski et al. 2006) we analyzed extended quintessence models, for which exact solutions of the Einstein equations are known, and discussed how in these models it is possible to treat the fine tuning problem in an alternative way. We applied our consideration to a special model, based on one of the most commonly used quintessence potentials $V(\phi)=\lambda \phi^{4}$, corresponding to the coupling $F(\phi)=(3 / 32) \phi^{2}$ (so-called induced gravity). We showed that this model corresponds to a special, and physically significant, case that emerged by requiring the existence of a Noether symmetry in the pointlike Lagrangian. In this paper we analyze a new and wider class of theories derived from the Noether symmetry requirement. One of the main advantages of such models is that they exhibit powerlaw couplings and potentials and admit a tracker behavior. In some sense we complete and generalize the analysis initiated in 
Marino \& de Ritis (2001) and de Ritis et al. (2000), where the attention was focused on the mechanism of obtaining an effective cosmological constant through the cosmological no-hair theorem, and the analysis of the solution was restricted to the asymptotical $t \rightarrow \infty$ regime. Extending our analysis to the whole time evolution, we are not only able to clarify the properties of such solutions, but also to compare predictions of these models with observations. We concentrate on the following data: the publicly available data on type Ia supernovae and gamma ray bursts, the parameters of large scale structure determined by the 2-degree Field Galaxy Redshift Survey (2dFGRS), and the measurements of cosmological distance with the Sunyaev-Zel'dovich effect.

\section{Model description}

\subsection{Specifying the model}

Since the detailed properties of a quintessence model, whose coupling and potential form are derived by requiring the existence of a Noether symmetry, are extensively discussed in Demianski et al. (2006, Paper I), here we only summarize the basic results, referring readers to our previous paper for details. Let us consider the general action for a scalar field $\phi$, non minimallycoupled with gravity, but not coupled with matter; in this case, we have

$\mathcal{A}=\int_{\mathrm{T}} \sqrt{-g}\left(F(\phi) R+\frac{1}{2} g^{\mu v} \phi_{, \mu} \phi_{, v}-V(\phi)+\mathcal{L}_{\mathrm{m}}\right) \mathrm{d}^{4} x$,

where $F(\phi)$ and $V(\phi)$ are two generic functions representing the coupling of the scalar field with geometry and its potential energy density respectively, $R$ is the scalar curvature, $\frac{1}{2} g^{\mu v} \phi_{, \mu} \phi_{, v}$ is the kinetic energy of the scalar field $\phi$, and $\mathcal{L}_{\mathrm{m}}$ describes the standard matter content. In units such that $8 \pi G_{\mathrm{N}}=\hbar=c=1$, where $G_{\mathrm{N}}$ is the Newtonian constant, we recover the standard gravity when $F$ is equal to $-1 / 2$, while in general the effective gravitational coupling is $G_{\text {eff }}=-\frac{1}{2 F}$. Here we would like to study the simple case of a homogeneous and isotropic universe, which implies that the scalar field $\phi$ only depends on time. It turns out that for the flat Friedman-Robertson-Walker universe filled with matter satisfying the equation of state $p=(\gamma-1) \rho$ and the scalar field $\phi$, the action in Eq. (1) reduces to the pointlike Lagrangian to

$\mathcal{L}=6 F a \dot{a}^{2}+6 F^{\prime} \dot{\phi} a^{2} \dot{a}+a^{3}\left(\frac{1}{2} \dot{\phi}^{2}-V(\phi)\right)-D a^{-3(\gamma-1)}$,

where $a$ is the scale factor and prime denotes derivative with respect to $\phi$, while the dot denotes derivative with respect to time. Moreover, the constant $D>0$ is defined in such a way that the matter density $\rho_{\mathrm{m}}$ is expressed as $\rho_{\mathrm{m}}=D\left(a_{o} / a\right)^{3 \gamma}$, where $1 \leq \gamma \leq 2$. The effective pressure and energy density of the $\phi$-field are given by

$p_{\phi}=\frac{1}{2} \dot{\phi}^{2}-V(\phi)-2(\ddot{F}+2 H \dot{F})$,

$\rho_{\phi}=\frac{1}{2} \dot{\phi}^{2}+V(\phi)+6 H \dot{F}$.

These two expressions, even if not pertaining to a conserved energy-momentum tensor, do define an effective equation of state $p_{\phi}=w_{\phi} \rho_{\phi}$, which drives the late time behavior of the model.

From now on we restrict ourselves to a dust-filled universe with $\gamma=1$, and $p_{\mathrm{m}}=0$. Using the point-like Lagrangian Eq. (2) in the action and varying it with respect to $\phi$, we obtain the EulerLagrange equations

$\ddot{\phi}+3 H \dot{\phi}+6\left(\dot{H}+2 H^{2}\right) F^{\prime}+V^{\prime}=0$,

$2 \dot{H}+3 H^{2}=\frac{1}{2 F}\left(p_{\phi}+p_{\mathrm{m}}\right)$,

together with the first integral

$H^{2}=-\frac{1}{6 F}\left(\rho_{\phi}+\rho_{\mathrm{m}}\right)$.

Let us now introduce the concept of an effective cosmological constant $\Lambda_{\text {eff. }}$. Using Eq. (7) it is natural to define the effective cosmological constant as $\Lambda_{\mathrm{eff}}=-\frac{\rho_{\phi}}{2 F}$ and the effective gravitational constant as $G_{\text {eff }}=-\frac{1}{2 F}$. With these definitions we can rewrite Eq. (7) as

$3 H^{2}=G_{\mathrm{eff}} \rho_{\mathrm{m}}+\Lambda_{\mathrm{eff}}$.

Introducing the standard Omega parameters by

$\Omega_{\mathrm{m}}=-\frac{\rho_{\mathrm{m}}}{6 F H^{2}}, \quad \Omega_{\Lambda_{\mathrm{eff}}}=\frac{\Lambda_{\mathrm{eff}}}{3 H^{2}}=-\frac{\rho_{\phi}}{6 F H^{2}}$,

we find as usual that

$\Omega_{\mathrm{m}}+\Omega_{\Lambda_{\mathrm{eff}}}=1$.

Imposing the Noether symmetry in the quintessence minisuperspace, $\{a, \phi ; \dot{a}, \dot{\phi}\}$, where the point-like Lagrangian is defined, it is possible to exactly integrate the Einstein field equations Eqs. (7) and (6), as well as to find a form for the two unknown functions $F(\phi)$ and $V(\phi)$ ) (for details see, Capozziello et al. 1996; Demianski et al. 1991). The existence of this symmetry actually leads to the following relation between the functions $F(\phi)$ and $V(\phi)$ :

$V=V_{0}(F(\phi))^{p(s)}$,

where $V_{0}$ is a constant, and

$p(s)=\frac{3(s+1)}{2 s+3}$

with $s$ a real number. Moreover, a possible simple choice for the coupling is

$F=\xi(s)\left(\phi+\phi_{0}\right)^{2}$,

where $\phi_{0}$ is a constant that does not affect our results and, therefore, from now on we set it to zero, and

$\xi(s)=\frac{(2 s+3)^{2}}{48(s+1)(s+2)}$.

Let us note that the form of the coupling given by (13) is quite relevant from the point of view of fundamental physics. It describes so-called induced gravity. The induced gravity model was initially proposed by Zee in 1979, as a theory of gravity incorporating the concept of spontaneous symmetry breaking. It was based on the observation that in gauge theories the dimensional coupling constants arising in a low-energy effective theory can be expressed in terms of the vacuum expectation values of scalar fields. In such a model the gravitational and cosmological constants are not introduced by hand, but are generated in the process of symmetry breaking of a scalar field non minimallycoupled with the Ricci scalar in the Lagrangian describing the 
system. Once the Noether symmetry is specified, it is possible to find a corresponding conserved quantity and use it as a new dynamical variable (for details see Capozziello et al. 1996; Demianski et al. 1991). One can then solve the corresponding Lagrange equations, and finally after returning to the original variables we obtain the sought-after $a(t)$ and $\phi(t)$. The final results can be written in the form

$a(t)=A(s)\left(B(s) t^{\frac{3}{s+3}}+\frac{D}{\Sigma_{0}}\right)^{\frac{s+1}{s}} t^{\frac{2 s^{2}(6 s+3}{s(s+3)}}$,

$\phi(t)=C(s)\left(-\frac{V_{0}}{\gamma(s)} B(s) t^{\frac{3}{s+3}}+\frac{D}{\Sigma_{0}}\right)^{-\frac{2 s+3}{2 s}} t^{-\frac{(2 s s+3)^{2}}{2 s(s+3)}}$,

where $A(s), B(s), C(s), \gamma(s)$, and $\chi(s)$ are given by

$A(s)=(\chi(s))^{\frac{s+1}{s}}\left(\frac{(s+3) \Sigma_{0}}{3 \gamma(s)}\right)^{\frac{s+2}{s+3}}$,

$B(s)=\left(\frac{(s+3)^{2}}{s+6}\right)\left(\frac{(s+3) \Sigma_{0}}{3 \gamma(s)}\right)^{-\frac{3}{(s+3)}}$,

$C(s)=(\chi(s))^{-\frac{(2 s+3)}{2 s}}\left(\frac{(s+3) \Sigma_{0}}{3 \gamma(s)}\right)^{-\frac{(3+2 s)}{2(s+3)}}$,

and

$\gamma(s)=\frac{2 s+3}{12(s+1)(s+2)}$,

$\chi(s)=-\frac{2 s}{2 s+3}$,

where $D$ is the matter density constant, $\Sigma_{0}$ a constant of integration resulting from the Noether symmetry, and $V_{0}$ the constant that determines the scale of the potential. Together with the independent parameters, we then use these three constants $\left(D, \Sigma_{0}, V_{0}\right)$, which however are not directly measurable, but they do parametrize the possible solutions of the model. In the next section we shall reduce their number by means of additional assumptions along the lines of Paper I.

\section{The parameter space}

As is apparent from Eqs. (15) and (16) there are two additional particular values of $s$, namely $s=0$ and $s=-3$, which should be treated independently.

When $s=0$, a Noether symmetry exists if

1. $F=F_{0} \phi^{2}$, and $V=V_{0} \phi^{2}$,

2. $F=-\frac{1}{2}$ (minimal coupling), and $V=V_{0}\left(A \mathrm{e}^{\mu \phi}-B \mathrm{e}^{-\mu \phi}\right)^{2}$, with $\mu=\sqrt{3 / 2}$, and $A, B$ being constants.

The case of the minimal coupling has been thoroughly investigated in Rubano \& Scudellaro (2002), Rubano et al. (2003), and Demianski et al. (2005). If $B=0$ we obtain an exponential potential, which is a very important model of quintessence with a standard scalar field. When $s=-3$, we recover the case of the quartic potential treated in the Paper I. We therefore concentrate on the other values of $s$. As we shall see in a moment, this will lead to a very different class of potentials from those discussed in Paper I. In fact we obtain inverse power-law type potentials, which are interesting and recently widely used in the literature.

First of all, we have to find the physically acceptable range for $s$, and the most important requirement is, of course,

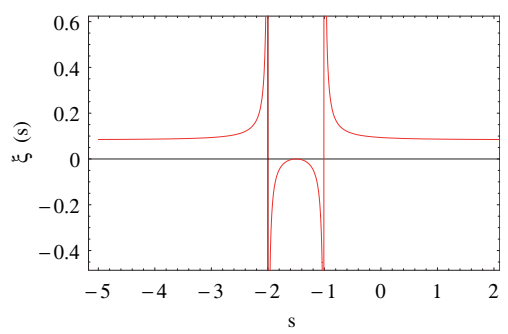

Fig. 1. Diagram of $\xi(s)$ as function of $s$. It turns out that an attractive gravity requires $s \in(-2,-1)$.

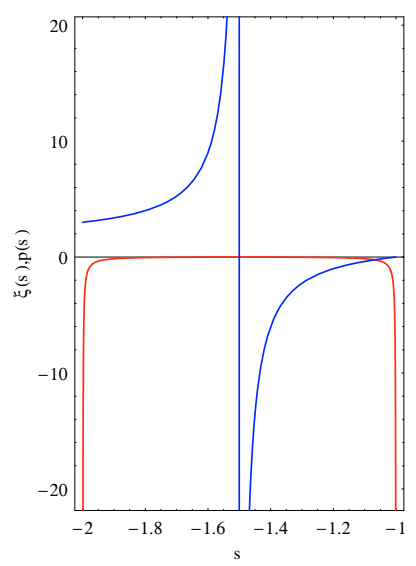

Fig. 2. Behavior of the coupling factor $\xi(s)$ (red curve) and the powerlaw exponent $p(s)$ (blue curve). We see that with an appropriate choice of $\mathrm{s}$ in the range $(-1.5,-1)$ all the values for the exponents are available.

that $G_{\text {eff }}>0$, i.e. $F<0$. This restricts $s$ to $(-2,-1)$ as shown in Fig. 1. As mentioned above in the range $s \in\left(-\frac{3}{2},-1\right)$ the potential $V(\phi)$ is of an inverse power-law type, $\phi^{-2|p(s)|}$. In this case our model naturally admits cosmological scaling solutions, recently studied, for example by Amendola (1999) and Uzan (1999) in the context of quintessence models. In Fig. 2 we see that all the possible exponents for the inverse power-law potential are available. To determine the integration constants $D$ and $\Sigma_{0}$, we follow the procedure used in Paper I, by using the age of the universe, $t_{0}$, as a unit of time. Because of our choice of time unit, the expansion rate $H(t)$ is dimensionless, so that our Hubble constant is clearly of order 1 and not (numerically) the same as the $H_{0}$ that is usually measured in $\mathrm{km} \mathrm{s}^{-1} \mathrm{Mpc}^{-1}$. Setting $a_{0}=a\left(t_{0}\right)=1$ and $\widehat{H}_{0}=H\left(t_{0}\right)$, we are able to express $\Sigma_{0}$ and $D$ as functions of $s$ and $\widehat{H}_{0}$. We obtain

$$
\begin{aligned}
D= & \left(\left(\frac{1}{A(s)}\right)^{\frac{s}{s+1}}-B(s)\right) \Sigma_{0} \\
\Sigma_{0}= & \left(3^{-\frac{5 s+6}{s^{2}+4 s+3}}(s+3)^{-\frac{3 s^{2}+7 s+3}{s^{2}+4 s+3}}(s+6) \gamma(s)\right)^{\frac{(s+1)(s+3)}{s^{2}-s-3}} \\
& \times\left(\frac{\left(\widehat{H}_{0}-2\right) s^{2}+3\left(\widehat{H}_{0}-2\right) s-3}{(s+1) \chi(s)}\right)^{\frac{(s+1)(s+3)}{s^{2}-s-3}} .
\end{aligned}
$$

Therefore, our family of models, labeled by $s$, depends only on $V_{0}$, and $\widehat{H}_{0}$. For both these parameters, we not only have a thorough knowledge of their physical meaning, but for $\widehat{H}_{0}$ we can also strongly constrain its range of variability. Actually, we may easily obtain the relation

$h=9.9 \frac{\widehat{H}_{0}}{\tau}$ 


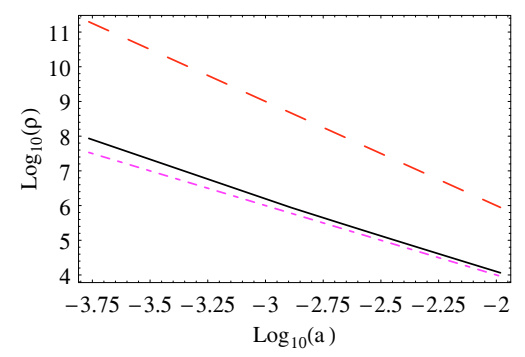

Fig. 3. Plot of $\log _{10} \rho_{\phi}$ versus $\log _{10} a$ (solid black line). The upper and lower dashed lines indicate the $\log -\log$ plot of $a^{-3}$ and $a^{-4}$ versus $a$, respectively. It turns out that $\rho_{\phi}$ scales as $a^{-n}$, with $3<n<4$. In this and subsequent plots, we use the mean values for the parameters obtained through fits (see Table 2).

where as usual $h=H_{0} / 100$ and $\tau$ is the age of the universe in Gy. We see that $\widehat{H}_{0}$ fixes only the product $h \tau$. In particular, we know that $\tau=13.73_{-0.15}^{+0.16}$ (see for instance Spergel et al. 2006), thus we get $h<0.76$ for $\widehat{H}_{0} \approx 1$. The actual value of $h$ may be obtained by some of the subsequent tests or in others it has to be set as a prior condition - this will be specified in each case considered below.

Using the available observational data, we can further constrain the range of possible values of $s$. Actually requiring that today $\ddot{a}\left(t_{0}\right)>0$, as indicated by observations of supernovae Ia and WMAP, we constrain the range of possible values of $s$ to $s \in(-1.5,-1.2)$.

From Eqs. (15) and (16) it turns out that for low values of $t$ the scale factor and the scalar field behave as

$a \propto t^{\frac{2 s^{2}+6 s+3}{s(s+3)}}$,
$\phi \propto t^{-\frac{(2 s+3)^{2}}{2 s(s+3)}}$.

Substituting these functions in $\rho_{\phi}$, as given by the Eq. (4), we get that for low $t$ the scalar field density $\rho_{\phi} \propto a^{-3}$. This is however true only asymptotically for very small $t$. Exact computation as shown in Fig. 3 gives $\rho_{\phi} \propto a^{-n}$, with $n \sim 3$. This justifies our assertion that our model naturally admits scaling solutions. The situation changes dramatically near the present time (see below). For high values of $t$, the scale factor and the scalar field behave as

$a \propto t^{\frac{2 s^{2}+9 s+6}{s(s+3)}}$,

$\phi \propto t^{-\frac{2 s+3}{s}}$,

respectively, as shown in Marino \& de Ritis (2001), where this asymptotical regime is discussed. It is interesting to note, as shown in Paper $\mathrm{I}$, that $w_{\phi}$ represents an equation of state, in the usual sense, of the effective cosmological constant $\Lambda_{\text {eff }}$, and that $\Lambda_{\text {eff }}$ asymptotically behaves as a true cosmological constant $\left(w_{\phi} \rightarrow-1\right)$ as $t \rightarrow \infty$. Since both $\rho_{\phi}$ and $p_{\phi}$ depend on $F(\phi)$ through its time derivative (see Eqs. (4) and (3)) and asymptotically $F(\phi) \sim$ constant, we recover in this limit the minimally coupled theory. In Figs. 4 and 5 we show the evolution with the redshift and the rate of evolution of $w_{\phi}$, where the fast transition toward $w_{\phi} \rightarrow-1$ appears between $z=3$ and $z=0.5$. Before reaching this asymptotic regime, the energy density $\rho_{\phi}$ is dominated by the coupling term $6 H \dot{F}$. Concluding this section we present the traditional plot $\log \rho_{\phi}-\log a$ and compare it with the evolution of matter density (see Fig. 6). It is interesting to see that $\rho_{\phi}$ follows the matter density during the matter-dominated era, and it becomes dominant at a late time.

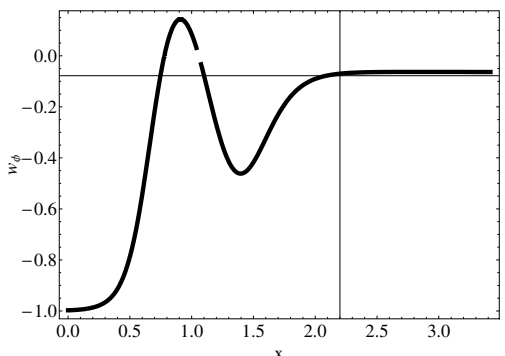

Fig. 4. $w_{\phi}$ as a function of $x=\log _{10}(1+z)$, for the averaged mean values provided by our analysis, as shown in Table 2 . We observe a transition from a small constant value in the past, $\left|w_{\phi}\right| \approx 0$, to $w_{\phi}=-1$ at present.

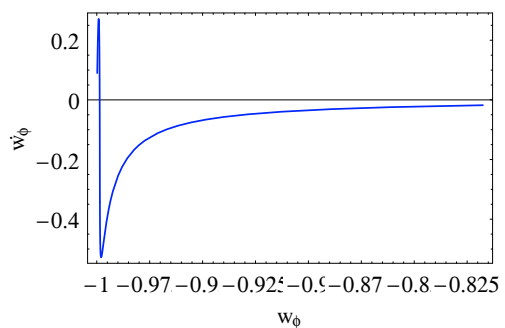

Fig. 5. Rate of change in the equation of state as measured by $\dot{w}_{\phi}$ versus the $w_{\phi}$ parameter. The values of the parameters correspond to the average values provided by our analysis and shown in Table 2 .

\section{Newtonian limit and parametrized post-Newtonian (PPN) behavior}

Recently, the cosmological relevance of extended gravity theories as scalar tensor or higher order theories has been widely explored. However, in the weak field approximation, all these classes of theories are expected to reproduce the Einstein general relativity that, in any case, is experimentally tested only in this limit. This fact is a matter of debate, since several relativistic theories do not reproduce Einstein results at the Newtonian approximation but, in some sense, generalize them, giving rise, for example, to Yukawa-like corrections to the Newtonian potential, which could have interesting physical consequences. Moreover, in general, any relativistic theory of gravitation can yield corrections to the Newton potential (see for example, Will 1993), which in the post-Newtonian (PPN) formalism could furnish tests for such theory, mainly based on the Solar System experiments. In this section we want to discuss the Newtonian limit of our class of scalar-tensor theories of gravity, the induced gravity theories, and to study the parametrized post Newtonian (PPN) behavior of these theories. In particular, it turns out that the Newtonian limit depends on $\xi(s)$. Furthermore, we find a quadratic correction to the Newtonian potential strictly depending on the presence of the scalar-field potential, which acts as a cosmological constant.

\subsection{Newtonian limit}

In order to recover the Newtonian limit of our theory described by the action in Eq. (1), associated with the effective stressenergy tensor

$$
\begin{aligned}
T_{\mu \nu}^{(\mathrm{eff})}= & \frac{1}{F(\phi)}\left\{-\frac{1}{2} \phi_{; \mu} \phi_{; \nu}+\frac{1}{4} g_{\mu \nu} g^{\alpha \beta} \phi_{; \alpha} \phi_{; \beta}\right. \\
& \left.-\frac{1}{2} g_{\mu \nu} V(\phi)-g_{\mu \nu} \square F(\phi)+F(\phi)_{; \mu \nu}-\frac{1}{2} T_{\mu \nu}^{\mathrm{m}}\right\},
\end{aligned}
$$




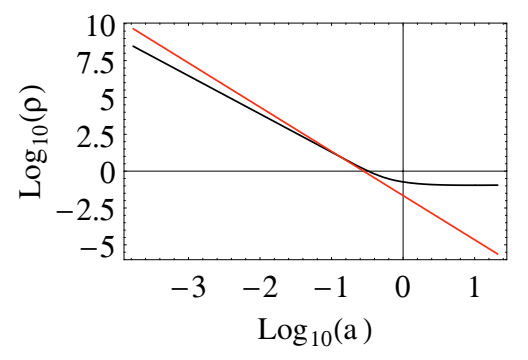

Fig. 6. Plot of $\log _{10} \rho_{\phi}$ versus $\log _{10} a$ in the Jordan frame. The vertical bar marks $\log _{10} a_{0}$. The solid red straight line indicates the log-log plot of $\rho_{\mathrm{m}}$ versus a. The matter dominated-era and the transition to the present dark-energy dominated regime are represented.

where $T_{\mu \nu}^{\mathrm{m}}$ is the usual stress-energy tensor of matter and $\square$ is the d'Alembert operator, we write the metric tensor as

$g_{\mu v}=\eta_{\mu v}+h_{\mu v}$,

where $\eta_{\mu \nu}$ is the Minkowski metric and $h_{\mu \nu}$ is a small correction. In the same way, we define the scalar field $\psi$ as a perturbation, of the same order as $h_{\mu \nu}$, of the original field $\phi$, that is

$\phi=\varphi_{0}+\psi$,

where $\varphi_{0}$ is a constant of order unity. It is clear that for $\varphi_{0}=1$ and $\psi=0$ the standard Einstein general relativity with $\Lambda$ is recovered. To write the Einstein tensor $G_{\mu \nu}$ in an appropriate form, we define the auxiliary fields

$\bar{h}_{\mu v} \equiv h_{\mu v}-\frac{1}{2} \eta_{\mu v} h$

and

$\sigma_{\alpha} \equiv \bar{h}_{\alpha \beta, \gamma} \eta^{\beta \gamma}$

where $h=\eta^{\mu v} h_{\mu v}$. Given these definitions, to the first order in $h_{\mu \nu}$, we obtain

$G_{\mu \nu}=-\frac{1}{2}\left\{\square_{\eta} \bar{h}_{\mu \nu}+\eta_{\mu \nu} \sigma_{\alpha, \beta} \eta^{\alpha \beta}-\sigma_{\mu, \nu}-\sigma_{v, \mu}\right\}$,

where $\square_{\eta} \phi \equiv \eta^{\mu v} \phi_{, \mu v}$. To obtain the weak-field limit of the field equations we also have to expand the effective stress-energy tensor. This means that it is necessary to expand the coupling function and the self-interacting potential. Specifically, it turns out that expanding the coupling function $F(\phi)$ and the selfinteracting potential $V(\phi)$ (by using their explicit forms) up to the second order in $\psi$, we get

$F(\phi)=\xi(s)\left(\varphi_{0}^{2}+2 \varphi_{0} \psi+\psi^{2}\right)$,

$$
\begin{aligned}
V(\phi) \simeq & \bar{V}_{0}\left(\varphi_{0}^{p(s)}+p(s) \varphi_{0}^{p(s)-1} \psi\right. \\
& \left.+\frac{p(s)(p(s)-1)}{2} \varphi_{0}^{p(s)-2} \psi^{2}+\cdots\right),
\end{aligned}
$$

where $\bar{V}_{0}=V_{0} \xi(s)^{p(s)}$. Then, to the first order, the effective stress-energy tensor becomes

$\tilde{T}_{\mu \nu}=-2 \varphi_{0}^{3} \eta_{\mu \nu} \square_{\eta} \psi+2 \varphi_{0}^{3} \psi_{, \mu \nu}-\frac{\bar{V}_{0} \varphi_{0}^{2+p(s)}}{2 \xi(s)} \eta_{\mu \nu}-\frac{1}{2} \frac{\varphi_{0}^{2}}{\xi(s)} T_{\mu \nu}^{\mathrm{m}}$ and the field equations assume the form

$$
\begin{aligned}
& \frac{1}{2}\left\{\square_{\eta} \bar{h}_{\mu \nu}+\eta_{\mu \nu} \sigma_{\alpha, \beta} \eta^{\alpha \beta}-\sigma_{\mu, v}-\sigma_{\nu, \mu}\right\}=2 \varphi_{0}^{3} \eta_{\mu \nu} \square_{\eta} \psi-2 \varphi_{0}^{3} \psi_{, \mu \nu} \\
& +\frac{\bar{V}_{0} \varphi_{0}^{2+p(s)}}{2 \xi(s)} \eta_{\mu \nu}+\frac{1}{2} \frac{\varphi_{0}^{2}}{\xi(s)} T_{\mu \nu}^{\mathrm{m}} .
\end{aligned}
$$

When $T_{\mu \nu}^{\mathrm{m}}$ describes a point particle of mass $M$ and for $p(s) \neq 4$ and $p(s) \neq 1$, we get

$$
\begin{aligned}
h_{00} \simeq & {\left[\varphi_{0}^{2} \frac{1-16 \xi(s)}{2 \xi(s)(1-12 \xi(s))}\right] \frac{M}{r} } \\
& -\left[\frac{M \varphi_{0}^{2}}{1-12 \xi(s)} \frac{\bar{V}_{0}(p(s)-4)(p(s)-1)}{1-2 \xi(s)}\right] r \\
& -4 \pi\left[\frac{\bar{V}_{0} \varphi_{0}^{2+p(s)}}{\xi(s)}+\frac{2 \varphi_{0}^{4}}{2(p(s)-1)} \frac{\bar{V}_{0}(p(s)-4)(p(s)-1)}{1-2 \xi(s)}\right] r^{2}, \\
h_{i j} \simeq & \delta_{i j}\left\{\left[\varphi_{0}^{2} \frac{1-8 \xi(s)}{2 \xi(s)(1-12 \xi(s))}\right] \frac{M}{r}\right. \\
& +\left[\frac{M \varphi_{0}^{2}}{1-12 \xi(s)} \frac{\bar{V}_{0}(p(s)-4)(p(s)-1)}{1-2 \xi(s)}\right] r \\
& \left.+4 \pi\left[\frac{\bar{V}_{0} \varphi_{0}^{2+p(s)}}{\xi(s)}-\frac{2 \varphi_{0}^{4}}{2(p(s)-1)} \frac{\bar{V}_{0}(p(s)-4)(p(s)-1)}{1-2 \xi(s)}\right] r^{2}\right\},
\end{aligned}
$$

where only terms linear in $V_{0}$ are given and we omitted the constant terms.

\subsection{Constraints on PPN parameters}

A satisfactory description of the PPN limit for scalar tensor theories is developed in Esposito-Farese (2004) and Damour et al. (1993). In these papers, this limit has been thoroughly discussed leading to interesting results even in the case of strong gravitational sources like pulsars and neutron stars where the deviations from general relativity are considered in a non-perturbative regime (Damour et al. 1993). The starting point for such an analysis is a redefinition of the non minimally-coupled Lagrangian action in terms of a minimally-coupled scalar field model via a conformal transformation of the form $\tilde{g}_{\mu v}=-2 F(\phi) g_{\mu v}$. In fact, assuming the transformation rules

$\left(\frac{\mathrm{d} \widetilde{\phi}}{\mathrm{d} \phi}\right)^{2}=\frac{3}{2}\left(\frac{\mathrm{d} \ln F(\phi)}{\mathrm{d} \phi}\right)^{2}-\frac{1}{2 F(\phi)}$,

and

$\widetilde{V}(\phi)=\frac{V(\phi)}{4 F(\phi)^{2}}, \quad \widetilde{\mathcal{L}}_{\mathrm{m}}=\mathcal{L}_{\mathrm{m}} F^{-2}(\phi)$,

one rewrites the action as

$\widetilde{\mathcal{A}}=\int \sqrt{-\widetilde{g}}\left[\widetilde{R}+\frac{1}{2} \widetilde{g}^{\mu v} \widetilde{\phi}_{, \mu} \widetilde{\phi}_{, v}-\widetilde{V}(\widetilde{\phi})+\widetilde{\mathcal{L}}_{\mathrm{m}}\right] \mathrm{d}^{4} x$.

The first consequence of such a transformation is that the nonminimal coupling is transferred to the ordinary matter sector, introducing an interaction term between matter and the scalar field. Actually, the Lagrangian $\widetilde{\mathcal{L}}_{\mathrm{m}}$ depends not only on the conformally transformed metric $\widetilde{g}_{\mu \nu}$ and the matter fields, but it also depends on the coupling function. In the same way, the field equations can be recast in the Einstein frame. The energy-momentum tensor is defined as $\widetilde{T}_{\mu \nu}^{\mathrm{m}}=\frac{2}{\sqrt{-\widetilde{g}}} \frac{\delta \widetilde{\mathcal{L}}_{\mathrm{m}}}{\delta \widetilde{g}_{\mu \nu}}$ and is related to the Jordan 
Table 1. A brief summary of recent constraints on the PPN-parameters.

\begin{tabular}{l|c}
\hline \hline Mercury Perih. Shift $^{2}$ & $\left|2 \gamma_{0}^{\mathrm{PPN}}-\beta_{0}^{\mathrm{PPN}}-1\right|<3 \times 10^{-3}$ \\
\hline Lunar Laser Rang. $^{3}$ & $4 \beta_{0}^{\mathrm{PPN}}-\gamma_{0}^{\mathrm{PPN}}-3=-(0.7 \pm 1) \times 10^{-3}$ \\
Very Long Bas. Int. $^{4}$ & $\left|\gamma_{0}^{\mathrm{PPN}}-1\right|=4 \times 10^{-4}$ \\
Cassini spacecraft $^{5}$ & $\gamma_{0}^{\mathrm{PPN}}-1=(2.1 \pm 2.3) \times 10^{-5}$ \\
\hline
\end{tabular}

References: ${ }^{2}$ Shapiro (1993); ${ }^{3}$ Williams et al. (1996); ${ }^{4}$ Shapiro et al. (2004); and ${ }^{5}$ Bertotti et al. (2003).

expression as $\widetilde{T}_{\mu \nu}^{\mathrm{m}}=\sqrt{-2 F(\phi)} T_{\mu \nu}^{\mathrm{m}}$. Possible deviations from the standard general relativity can be tested through the Solar System experiments (Will 1993) and binary pulsar observations, which give an experimental estimate of the PPN parameters. The generalization of these quantities to the scalar-tensor theories allows the PPN-parameters to be expressed in terms of the nonminimal coupling function $F(\phi)$, and in our case using Eqs. (13) and (14), we obtain

$\gamma^{\mathrm{PPN}}-1=-\frac{F^{\prime}(\phi)^{2}}{F(\phi)+2\left[F^{\prime}(\phi)\right]^{2}}=-\frac{4 \xi(s)}{1+8 \xi(s)}$,

$\beta^{\mathrm{PPN}}-1=\frac{F(\phi) \cdot F^{\prime}(\phi)}{F(\phi)+3\left[F^{\prime}(\phi)\right]^{2}} \frac{\mathrm{d} \gamma^{\mathrm{PPN}}}{\mathrm{d} \phi}=0$.

The above definitions imply that the PPN-parameters in general depend on the non-minimal coupling function $F(\phi)$ and its derivatives. However in our model $\gamma^{\text {PPN }}$ depends only on $s$ while $\beta^{\text {PPN }}=1$. The PPN-parameters can be directly constrained by the observational data. Actually, Solar System experiments give accurate indications on the ranges of ${ }^{1} \gamma_{0}^{\mathrm{PPN}}, \beta_{0}^{\mathrm{PPN}}$.

We summarize the experimental results in Table 1 . These results have been used by Schimd et al. (2005) to set the following constrains:

$\left|\gamma_{0}^{\mathrm{PPN}}-1\right| \leq 2 \times 10^{-3}, \quad\left|\beta_{0}^{\mathrm{PPN}}-1\right| \leq 6 \times 10^{-4}$.

It turns out that the limit for $\beta_{0}^{\mathrm{PPN}}$ in the Eq. (46) is naturally verified, for each value of $s$, while the constraint on $\left|\gamma_{0}^{\mathrm{PPN}}-1\right|$ is satisfied only for $s \in(-1.5,-1.4)$, as shown in Fig. 7 .

For the sake of completeness, here we even take into account the shift that the scalar-tensor gravity induces on the theoretical predictions for the local value of the gravitational constant as coming from the Cavendish-like experiments. This quantity represents the gravitational coupling measured when the Newton force arises between two masses:

$G_{\text {Cav }}=\frac{F \cdot r^{2}}{m_{1} \cdot m_{2}}$.

In the case of scalar tensor gravity, the Cavendish coupling is related to $F$ and $F^{\prime}$ and is given by:

$$
\begin{aligned}
G_{\mathrm{Cav}} & =-\frac{1}{2 F(\phi)}\left[1+\frac{\left[2 F^{\prime}(\phi)\right]^{2}}{4 F(\phi)+3\left[2 F^{\prime}(\phi)\right]^{2}}\right] \\
& =-\frac{1}{2 \xi(s) \phi^{2}}\left[\frac{1+16 \xi(s)}{1+12 \xi(s)}\right]=G_{\mathrm{eff}}\left[\frac{1+16 \xi(s)}{1+12 \xi(s)}\right],
\end{aligned}
$$

and in our models it depends only on $s$.

\footnotetext{
${ }^{1}$ We indicate with the subscript ${ }_{0}$ that the estimates are based on Solar System observations.
}

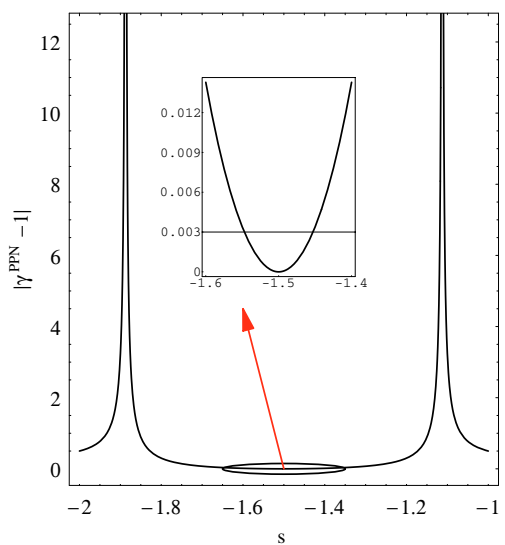

Fig. 7. Current limits on the PPN parameters restrict the range of the parameter $s$. We see that the constraint on $\left|\gamma_{0}^{\mathrm{PPN}}-1\right|<2 \times 10^{-3}$ leads to $s \in(-1.5,-1.4)$, as shown in the inner zoom.

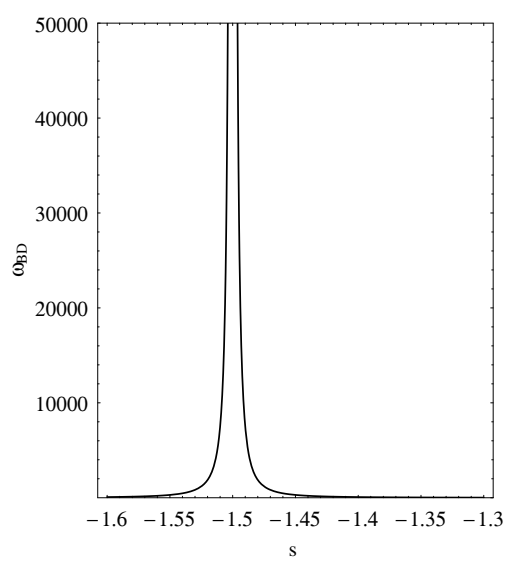

Fig. 8. Behavior of the Brans-Dicke parameter $\omega_{\mathrm{BD}}$ as a function of $s$. For $s \in(-1.6,-1.3), \omega_{\mathrm{BD}}$ satisfies limits placed by the solar system experiments $\left(\omega_{\mathrm{BD}}>40000\right)$ and by current cosmological observations $\left(\omega_{\mathrm{BD}}>120\right)$.

Finally, in Fig. 8 we plot the Brans-Dicke parameter $\omega_{\mathrm{BD}}$ as a function of $s$ : actually, for our model $\omega_{\mathrm{BD}}=-\frac{F(\phi)}{2 F^{\prime}(\phi)^{2}}=-\frac{1}{4 \xi(s)}$. It turns out that just for $s \in(-1.5,-1.4), \omega_{\mathrm{BD}}$ satisfies the limits coming both from the Solar System experiments, $\omega_{\mathrm{BD}}>40000$ (Will 1993), and current cosmological observations, including cosmic microwave anisotropy data and the galaxy power spectrum data, give $\omega_{\mathrm{BD}}>120$ (Acquaviva et al. 2005) ${ }^{2}$.

\section{Some considerations about conformal transformations and interacting dark energy}

Conformal transformations are often used to convert the non minimally-coupled scalar field models into the

2 It is worth noting that the legitimacy of the procedure of direct comparison of local and cosmological observations, in order to estimate variations of the physical constants, is a rather strong assumption, which deserves a proof or at least a justification. There is actually no reason a priori why local experiments should reveal variations occurring on cosmological scales, and in regions which are participating in the Hubble expansion. This interesting aspect of scalar tensor theories of gravity is discussed in Clifton et al. (2005) and in Shaw \& Barrow (2005), where it is shown that such procedure is correct. 
minimally-coupled ones, to gain mathematical simplification. The Jordan frame, in which the scalar field is non minimallycoupled to the Ricci curvature, is mapped into the Einstein frame in which the transformed scalar field is minimally-coupled but at a price of coupling matter to the scalar field. The two frames are not physically equivalent, and some care has to be taken in applying this technique (see for instance Faraoni 2000 for a critical discussion of this point). In this section we study the effect of conformal transformations on our models and show that, in presence of matter, it can mimic a coupling between the quintessence scalar field and dark matter. We discuss some implications of such a fictitious interaction on the effective equation of state. Actually it turns out that, since the interaction alters the redshift dependence of the matter density, it is possible to obtain an effective transformed dark-energy equation of state of $\widetilde{w}_{\text {eff }}<-1$. Let us start from the transformation rules connected with the conformal transformation $\widetilde{g}_{\mu \nu}=-2 F(\phi) g_{\mu \nu}$ :

$$
\begin{aligned}
\frac{\mathrm{d} \widetilde{\phi}}{\mathrm{d} \phi} & =\sqrt{\frac{3 F^{\prime}(\phi)^{2}-F(\phi)}{2 F(\phi)^{2}},} \\
\widetilde{a} & =\sqrt{-2 F(\phi)} a, \\
\frac{\mathrm{d} \widetilde{t}}{\mathrm{~d} t} & =\sqrt{-2 F(\phi)}, \\
\widetilde{V}(\phi) & =\frac{V(\phi)}{4 F(\phi)^{2}} .
\end{aligned}
$$

With these new variables the Lagrangian in the Eq. (2) becomes

$$
\widetilde{\mathcal{L}}=3 \widetilde{a}_{\vec{a}}^{2}+\widetilde{a}^{3}\left(\frac{1}{2} \dot{\bar{\phi}}^{2}-\widetilde{V}(\widetilde{\phi})\right)+\widetilde{\mathcal{L}}_{\mathrm{m}},
$$

where the dot now denotes derivative with respect to $\widetilde{t}$, and

$\widetilde{\mathcal{L}}_{\mathrm{m}}(\widetilde{\phi}, \widetilde{a})=\sqrt{-2 F(\phi)} \mathcal{L}_{\mathrm{m}}=\sqrt{-2 F(\phi)} D a^{-3(\gamma-1)}$.

An observer in the Einstein frame would infer that the scalar field is coupled to the dark matter, and this interaction is represented by the term $f(\widetilde{\phi}(\phi))=\sqrt{-2 F(\phi)}$, as can be seen more clearly from the field equations:

$$
\begin{aligned}
\ddot{\widetilde{\phi}}+3 \widetilde{H} \dot{\bar{\phi}} & =-\widetilde{V}_{\widetilde{\phi}}-\frac{\rho_{\mathrm{m}_{0}}}{\widetilde{a}^{3}} \frac{f_{\widetilde{\phi}}}{f\left(\widetilde{\phi}_{0}\right)}, \\
3 \widetilde{H}^{2} & =\left(\frac{1}{2} \dot{\widetilde{\phi}}^{2}-\widetilde{V}(\widetilde{\phi})\right)+f(\widetilde{\phi}) \frac{\rho_{\mathrm{m}_{0}}}{f\left(\widetilde{\phi}_{0}\right)} \widetilde{a}^{-3},
\end{aligned}
$$

where $\rho_{\mathrm{m}_{0}}$ is the actual matter density (treated here as dust), $\widetilde{\phi}_{0}$ the value of $\widetilde{\phi}$ today, and $\frac{\rho_{\mathrm{m}_{0}}}{f\left(\bar{\phi}_{0}\right)} \equiv D$. It turns out that the density of dark matter does not evolve as $\widetilde{a}^{-3}$, but scales as $\widetilde{\rho}_{\mathrm{m}} \propto \frac{f}{\widetilde{a}^{3}}$. Also the Klein-Gordon Eq. (55) differs from the standard one because of the last term on the right hand side. As shown in (Das et al. 2006) interaction between dark matter and dark energy could result in an effective equation of state $\widetilde{p}_{\text {eff }}=\widetilde{w}_{\text {eff }} \widetilde{\rho}_{\text {eff }}$, mimicking the $\Lambda C D M$ model. Actually $\widetilde{w}_{\text {eff }}$ is defined by the matter continuity equation

$$
\frac{\mathrm{d} \widetilde{\rho}_{\mathrm{eff}}}{\mathrm{d} \widetilde{t}}=-3 \widetilde{H}\left(1+\widetilde{w}_{\mathrm{eff}}\right) \widetilde{\rho}_{\mathrm{eff}},
$$

where

$\widetilde{\rho}_{\mathrm{eff}} \equiv \frac{\rho_{\mathrm{m}_{0}}}{\widetilde{a}^{3}}\left(\frac{f(\widetilde{\phi})}{f\left(\widetilde{\phi}_{0}\right)}-1\right)+\widetilde{\rho}_{\phi}$
It can be shown that $\widetilde{w}_{\text {eff }}$ is simply related to $\widetilde{w}_{\phi}$

$\widetilde{w}_{\mathrm{eff}}=\frac{\widetilde{w}_{\phi}}{1-x}$

where

$x \equiv-\frac{\rho_{\mathrm{m}_{0}}}{\widetilde{a}^{3} \widetilde{\rho}_{\phi}}\left(\frac{f(\widetilde{\phi})}{f\left(\widetilde{\phi}_{0}\right)}-1\right)$.

Since $x=0$ today, one has $\widetilde{w}_{\text {eff }}^{(0)}=\widetilde{w}_{\phi}^{(0)}$, which is greater than or equal to -1 . It turns out, however, that $f$ increases in time (Das et al. 2006), so that $x \geq 0$ and it is possible to have $\widetilde{w}_{\text {eff }}<-1$ in the past. In such a way interaction between dark matter and dark energy could generate a superquintessence regime, provided that the observer treats the dark matter as non-interacting, and ascribes part of the dark matter density to the scalar field, as is shown in Eq. (58). On the other hand an interacting dark energy component could hide the effect of a non standard gravity, in the Einstein frame, provided that it is considered as the physical one.

\subsection{Conformally transformed coupled dark energy}

Let us consider our nonminimally coupled model characterized by the functions $F(\phi)=\xi(s) \phi^{2}$ and $V(\phi)=V_{0}(F(\phi))^{p(s)}$. According to the rules in Eqs. (49)-(52) we obtain the following relations between the transformed and original dynamical quantities:

$$
\begin{aligned}
\widetilde{\phi} & =\sqrt{\frac{12 \xi(s)-1}{2 \xi(s)}} \ln \phi, \\
\widetilde{a} & =\sqrt{-2 \xi(s)} \phi a, \\
\widetilde{V} & =\frac{1}{4} V_{0} \xi(s)^{p(s)-2} \phi^{2 p(s)-4} .
\end{aligned}
$$

As we see from Eq. (62), the cosmic evolution is mediated in the transformed frame by the presence of the scalar field. The explicit form of Eq. (51), which connects the cosmic time in both frames, can be written in analytical form, but is indeed rather complicated, because actually it turns out that

$$
\begin{aligned}
\widetilde{t}= & \left\{2 C(s) s(s+3) t^{-\frac{2 s^{2}+6 s+9}{2(s+3) s}}\left[\left(\frac{1}{A(s)}\right)^{\frac{s}{s+1}}-B(s)-\frac{V_{0} B(s) t^{\frac{3}{s+3}}}{\gamma(s)}\right]^{1-\frac{3}{2 s}}\right. \\
& \left.\times\left(1-\frac{V_{0} B(s) t^{\frac{3}{s+3}}}{\left(\left(\frac{1}{A(s)}\right)^{\frac{s}{s+1}}-B(s)\right) \gamma(s)}\right)^{\frac{3}{2 s}} \gamma(s)\right\} \\
& \times \frac{{ }_{2} F_{1}\left[\frac{-2 s^{2}-6 s-9}{6 s}, 1+\frac{3}{2 s} ; \frac{-2 s^{2}-6 s-9}{6 s}+1 ; \frac{V_{0} B(s) t \frac{3}{s+3}}{\left[\left(\frac{1}{A(s)}\right)^{\frac{s}{s+1}}-B(s)\right] \gamma(s)}\right]}{\left(2 s^{2}+6 s+9\right)} \\
& \times \frac{1}{\left(\left(\frac{1}{A(s)}\right)^{\frac{s}{s+1}}-B(s)\right)\left(-\gamma(s)\left(\frac{1}{A(s)}\right)^{\frac{s}{s+1}}+V_{0} B(s) t^{\frac{3}{s+3}}+B(s) \gamma(s)\right)} .
\end{aligned}
$$

From Eqs. (61)-(64) we can evaluate the scalar field energy density and pressure, and the equation of state $\widetilde{w}_{\phi}$ according to the usual definitions. As in the Jordan frame, it turns out that also in the Einstein frame $\widetilde{w}_{\phi} \rightarrow-1$ for $\widetilde{t} \rightarrow \infty$ (see for instance Fig. 10).

Concluding this section we present the traditional plot $\log \widetilde{\rho}_{\phi}-\log \widetilde{a}$ and compare it with the $\log \rho_{\mathrm{m}}-\log a$ relation (see Fig. 11). Interestingly we see that, just because of the interaction term, $\widetilde{\rho}_{\phi}$ no longer tracks the matter during the matter 


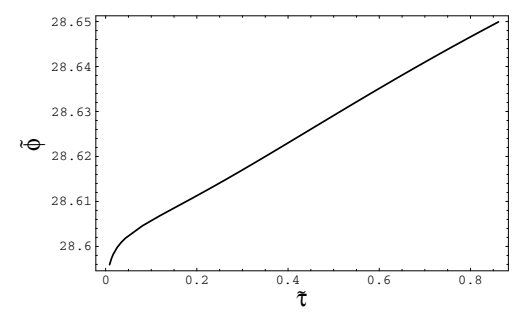

Fig. 9. Time evolution of the transformed scalar field $\widetilde{\phi}$.

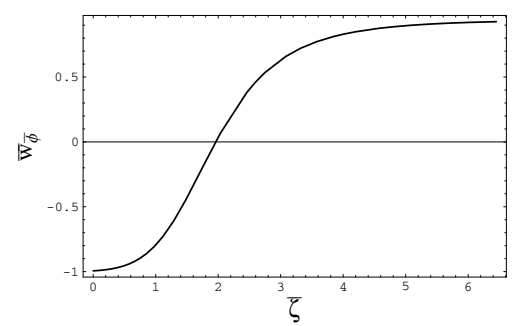

Fig. 10. Evolution with the redshift of $\widetilde{w}_{\phi}$ in the Einstein frame.

dominated era, as happens in the Jordan frame (see Fig. 6), but becomes dominant at earlier times. It is interesting to write down the effective equation of state $\widetilde{w}_{\text {eff }}$ (see Eq. (57)), which mimics a CDM model. Actually in our case, $\widetilde{w}_{\text {eff }}$ is

$\widetilde{w}_{\mathrm{eff}}=\frac{\widetilde{w}_{\phi}}{1-x} \equiv-\frac{\rho_{\mathrm{m}_{0}}}{\widetilde{a}^{3} \widetilde{\rho}_{\phi}}\left(\frac{\widetilde{\phi}+\widetilde{\phi}_{0}}{2 \widetilde{\phi}_{0}-1}\right)$.

It turns out that here $\widetilde{w}_{\text {eff }}$ and $\widetilde{w}_{\phi}$ are practically indistinguishable, since in our model the transformed scalar field $\widetilde{\phi}$ weakly evolves with time as shown in Fig. 9.

\subsection{Non minimally coupled quintessence and mass varying neutrinos through conformal transformations}

In this section we briefly discuss how the scalar tensor theories of gravity could be involved in a cosmological model with mass varying neutrinos that mimic the dark energy, a quite different theoretical scenario of evolution of the universe that recently has been suggested by Fardon et al. (2004). Let us recall that the mass differences between neutrino mass eigenstates $\left(m_{1}, m_{2}, m_{3}\right)$ have recently been measured in oscillation experiments (Lesgourgues \& Pastor 2006). Observations of atmospheric neutrinos suggest a squared mass difference of $\Delta m^{2} \sim 3 \times 10^{-3} \mathrm{eV}^{2}$, while solar neutrino observations and results from the KamLAND neutrino experiment point towards $\Delta m^{2} \sim 5 \times 10^{-5} \mathrm{eV}^{2}$. While only weak constraints on the absolute mass scale $\left(\Sigma m_{v}=m_{1}+m_{2}+m_{3}\right)$ have been obtained from single $\beta$-decay experiments, the double-beta decay searches from the Heidelberg-Moscow experiment have reported a signal for a neutrino mass at $>4 \sigma$ level (Klapdor-Kleingrothaus et al. 2004), recently promoted to $>6 \sigma$ level (Klapdor-Kleingrothaus 2006). This last result translates into a total neutrino mass of $\Sigma m_{v}>$ $1.2 \mathrm{eV}$ at $95 \%$ c.1., but this claim is still considered controversial (see Elliott \& Engel 2004).

It is known in the literature (Lesgourgues \& Pastor 2006) that massive neutrinos can be extremely relevant for cosmology as they leave key signatures in several cosmological data sets. More specifically, massive neutrinos suppress the growth of fluctuations on scales below the horizon scale when they become non relativistic. Current cosmological data have been able

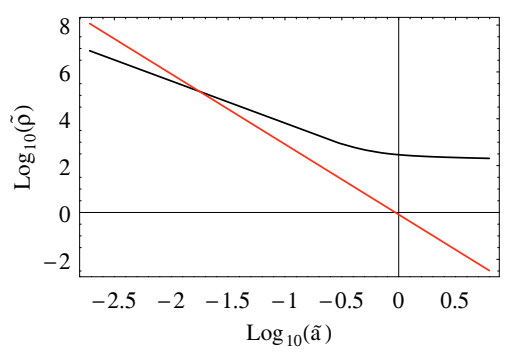

Fig. 11. Plot of $\log _{10} \widetilde{\rho}_{\phi}$ versus $\log _{10} \widetilde{a}$ in the Einstein frame. The vertical bar marks $\log _{10} \widetilde{a}_{0}$.

to indirectly constrain the absolute neutrino mass to $\Sigma m_{v}<$ $0.75 \mathrm{eV}$ at $95 \%$ c.l. (Spergel et al. 2006), and are challenging the Heidelberg-Moscow claim. However, as first noticed by (Hannestad 2005), there is some form of anticorrelation between the equation of state parameter $w$ and $\Sigma m_{v}$. The cosmological bound on neutrino masses can therefore be relaxed by considering a dark energy component with a more negative value of $w_{\mathrm{eff}}$ than a cosmological constant. Actually it has been proved that the Heidelberg-Moscow result is compatible with the cosmological data only if the equation of state (with $w$ being constant) is $w_{\text {eff }}<-1$ at $95 \%$.

This result suggests an interesting link between neutrinos and dark energy (see for instance Brookfield et al. 2006b,a; Bi et al. 2004; Kaplan et al. 2004; Amendola 2004). According to this scenario the late-time accelerated expansion of the universe is driven by the coupling between the quintessential scalar field and neutrinos. Because of this coupling the mass of neutrinos becomes a function of this scalar field. Since the scalar field evolves with time, the mass of neutrinos is not constant (massvarying neutrinos): the main theoretical motivation for this connection relies on the fact that the energy scale of the dark energy is close to the neutrinos mass scale. Moreover, as discussed above, in interacting dark energy models, the net effect of the interaction is to change the apparent equation of state of the dark energy, allowing a so-called superquintessence regime, with $w_{\text {eff }}<-1$. Interestingly enough, if the Heidelberg-Moscow results are combined with the WMAP 3-years data and other independent cosmological observations, such as the ones connected with the large-scale structure - coming from galaxy redshift surveys and Lyman- $\alpha$ forests - or with the SNIa surveys, it is possible to constrain the equation of state to $-1.67<w_{\text {eff }}<-1.05$ at $95 \%$ c.l., ruling out a cosmological constant greater than $95 \%$ c.l. (see De La Macorra et al. 2007).

In the following we discuss the coupling between neutrinos and dark energy from the point of view of the conformal transformation, according to the arguments outlined in the previous section; i.e. we show that the neutrino mass and scalar field coupling can be interpreted as an effect of conformal transformations from the Jordan to the Einstein frames, as it happens for the coupling between the dark energy and the dark matter. For our purpose the neutrinos can be either Dirac or Majorana particles, the only necessary ingredient is that, according to Fardon et al. (2004), the neutrino mass is a function of the scalar field. In the cosmological context, neutrinos should be treated as a gas (Brookfield et al. 2006b) and described by the collisionless distribution function $f\left(x^{i}, p^{i}, \tau\right)$ in the phase space (where $\tau$ is the conformal time) that satisfies the Boltzmann equation. When neutrinos are collisionless, the distribution function $f$ does not depend explicitly on time. We can then solve the Boltzmann 
equation and calculate the energy density stored in neutrinos $\left(f_{0}\right.$ is the background neutrino distribution function):

$\widetilde{\rho}_{v}=\frac{1}{\widetilde{a}^{4}} \int \widetilde{q}^{2} \mathrm{~d} \widetilde{q} \mathrm{~d} \Omega \varepsilon f_{0}(\widetilde{q})$,

with $\varepsilon^{2}=\widetilde{q}^{2}+m_{v}(\widetilde{\phi})^{2} \widetilde{a}^{2}, \widetilde{a}$ is the scale factor and $\widetilde{q}^{i}=\widetilde{a} \widetilde{p}^{i}$ is the comoving momentum. The pressure is

$\widetilde{p}_{v}=\frac{1}{3 \widetilde{a}^{4}} \int \widetilde{q}^{2} \mathrm{~d} \widetilde{q} \mathrm{~d} \Omega f_{0}(\widetilde{q}) \frac{\widetilde{q}^{2}}{\varepsilon}$.

From these equations, we derive that

$\dot{\tilde{\rho}}_{v}+3 \widetilde{H}\left(\widetilde{\rho}_{v}+\widetilde{p}_{v}\right)=\frac{\partial \ln m_{v}}{\partial \widetilde{\phi}} \dot{\bar{\phi}}\left(\widetilde{\rho}_{v}-3 \widetilde{p}_{v}\right)$,

(note that here the dot denotes the derivative with respect to $\tau$ ). The Klein Gordon equation for the scalar field reads

$\ddot{\widetilde{\phi}}+2 \widetilde{\tilde{H}} \dot{\bar{\phi}}+\widetilde{a}^{2} \frac{\partial \widetilde{V}}{\partial \widetilde{\phi}}=-\widetilde{a}^{2} \frac{\partial \ln m_{v}}{\partial \widetilde{\phi}}\left(\widetilde{\rho}_{v}-3 \widetilde{p}_{v}\right)$.

We see that Eq. (69) is formally equivalent to Eq. (55), but now the term on the righthand side describes coupling of the scalar field to the neutrino mass. As shown above, the interaction between neutrinos and dark energy could result in an effective equation of state $\widetilde{w}_{\text {eff }}$, defined in Eq. (57), while $\widetilde{w}_{\phi}$ is defined in the standard way by $\widetilde{p}_{\phi}=\widetilde{w}_{\phi} \widetilde{\rho}_{\phi}$. As in the previous section, the $\widetilde{w}_{\text {eff }}$ parameter is related to $\widetilde{w}_{\phi}$ by

$\widetilde{w}_{\mathrm{eff}}=\frac{\widetilde{w}_{\phi}}{1-\widetilde{x}}$

with

$\widetilde{x}=-\frac{\widetilde{\rho}_{\nu 0}}{\widetilde{a}^{3} \widetilde{\rho}_{\phi}}\left[\frac{m_{v}(\widetilde{\phi})}{m_{v}\left(\widetilde{\phi}_{0}\right)}-1\right]$.

Also in this case $\widetilde{w}_{\text {eff }}$ can be less than -1 , as was pointed out in the context of models with dark-matter/dark-energy interaction. This circumstance could lead to an observational test to establish which of the frames, Jordan or Einstein, is the physical one, since the coupling between the quintessential scalar field is provided by the function $F(\phi)$, which should drive not only the dark-matter evolution (see Eq. (55)), but also the neutrinos mass variation and the evolution of the gravitational constant $G_{\mathrm{eff}}=-\frac{1}{2 F}$.

\section{Observational data and predictions of our models}

\subsection{Constraints from recent SNla observations}

In this section we present results from fits of predictions of our model to the best SNIa data sets presently available. As a starting point we consider the sample of 182 SNIa compiled in Riess et al. (2007), which includes the 21 new type Ia supernovae recently discovered with the Hubble Space Telescope (HST), and combines previous SNIa data sets, namely the Gold Sample compiled in Riess et al. (2004) supplemented by the SNLS data set (Astier et al. 2005). Following the procedure described in Paper I, we perform a $\chi^{2}$ analysis comparing the redshift dependence of the theoretical values to the observational estimates of the distance modulus, $\mu=m-M$, which in scalar tensor theories of gravity takes the form

$m-M=5 \log D_{L}(z)+25+\Delta M_{G}$.

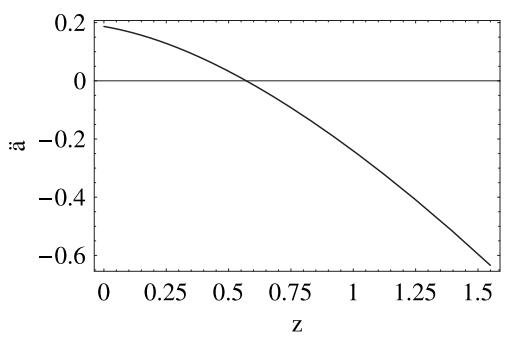

Fig. 12. Redshift dependence of the second derivative of the scale factor. The transition from a decelerating to an accelerating expansion occurs close to $z \sim 0.5$, as predicted by recent observations of SNIa $z_{t}=0.46 \pm$ 0.13 (Riess et al. 2004, 2007).

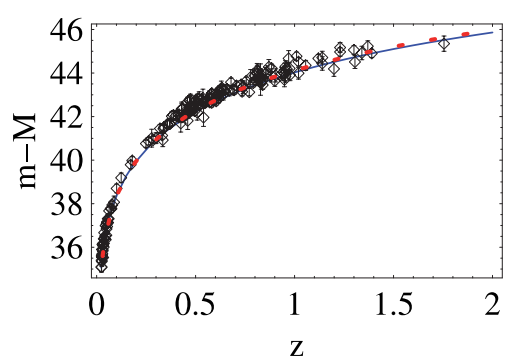

Fig. 13. Observational data of the SNIa sample compiled by Riess et al. (2007) fitted to our model. The solid curve is the best fit curve, compared with a standard $\Lambda \mathrm{CDM}$ model with $\Omega_{\Lambda}=0.71$ (red dashed line).

Here the presence of the correction term

$\Delta M_{G}=\frac{15}{4} \log \left(\frac{G_{\text {eff }}}{G_{\mathrm{eff}_{0}}}\right)$,

describes the effect of the time variation of the effective gravitational constant $G_{\text {eff }}$ on the luminosity of high-redshift supernovae and allows one to test the scalar tensor theories of gravity (Gaztañaga et al. 2001; Uzan 2003) using the SNIa data. Moreover, the luminosity distance for a general flat and homogeneous cosmological model can be expressed as an integral of the Hubble function as

$D_{L}(z)=\frac{c}{H_{0}}(1+z) \int_{0}^{z} \frac{1}{E(\zeta)} \mathrm{d} \zeta$

where $E(z)=\frac{H(z)}{H_{0}}$ is related to the Hubble function expressed in terms of $z=a_{0} / a(t)-1$. Let us note that the luminosity distance also depends on the Hubble distance $\frac{c}{H_{0}}$ (which does not depend on the choice of the unit of time). Such freedom allows us to fit $h$ or the a priori unknown age of the universe $\tau$ using the SNIa dataset. We find that $\chi_{\text {red }}^{2}=0.98$ for 182 data points, and the best fit values are $\widehat{H}_{0}=0.96_{-0.05}^{+0.05}, s=-1.46_{-0.03}^{+0.04}$, which corresponds to $\Omega_{\Lambda_{\text {eff }}}=0.69_{-0.06}^{+0.04}$. We also get $h=0.72 \pm 0.04$. In Fig. 13 we compare the best-fit curve with the observational data sets.

\subsubsection{Dimensionless coordinate distance test}

After having explored the Hubble diagram of SNIa, let us now follow the more general approach, as suggested by Daly \& Djorgovski (2004) and already tested in Paper I. Consider as a cosmological observable the dimensionless coordinate distance defined as

$y(z)=\int_{0}^{z} \frac{1}{E(\zeta)} \mathrm{d} \zeta$ 


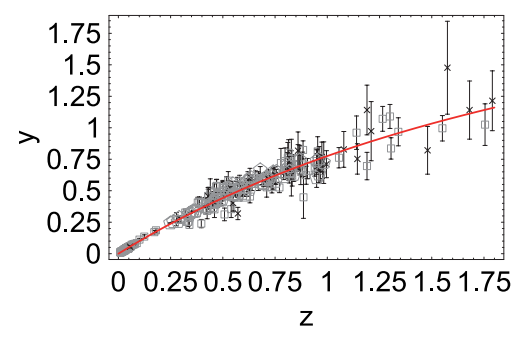

Fig. 14. Updated Daly \& Djorgovski database (Daly \& Djorgovski 2005) fitted to our model. The solid curve is the best fit curve with $\chi_{\text {red }}^{2}=1.1$ for 248 data points, and the best fit values are $\widehat{H}_{0}=.97_{-0.03}^{+0.04}$, $s=-1.49_{-0.04}^{+0.02}$.

noting that $y(z)$ does not explicitly depend on $h$, so that any choice for $h$ does not alter the main result. Daly \& Djorgovski have determined $y(z)$ for the SNIa in the Gold Sample of Riess et al. (2004) which is the most homogeneous SNIa sample available today. Since SNIa allows $D_{L}$ to be estimated rather than $y$, a value of $h$ has to be set. Fitting the Hubble law to a large set of low redshift $(z<0.1)$ SNIa, Daly \& Djorgovski (2004) have set

$h=0.66 \pm 0.08$.

To enlarge the sample, Daly \& Djorgovski added 20 points on the $y(z)$ diagram using a technique of distance determination based on the angular dimension of radiogalaxies (Daly \& Djorgovski 2004). This data set has been recently supplemented by 71 new supernovae from the Supernova Legacy Survey of Astier et al. (2005), which allowed determination of dimensionless coordinate distances to these supernovae. These were obtained using the values and uncertainties of $\mu_{B}$ listed in Table 9 of Astier et al. (2005), with $h=0.7$. This extended sample that spans the redshift range $(0.1,1.8)$ has been suitably homogenized. Using the following merit function

$\chi^{2}\left(s, \widehat{H}_{0}\right)=\frac{1}{N-3} \sum_{i=1}^{N}\left[\frac{y\left(z_{i} ; \alpha_{1}, \widehat{H}_{0}\right)-y_{i}}{\sigma_{i}}\right]^{2}$,

we find that $\chi_{\text {red }}^{2}=1.1$ for 248 data points, and the best-fit values are $\widehat{H}_{0}=.97_{-0.03}^{+0.04}, s=-1.49_{-0.04}^{+0.02}$. In Fig. 14 we compare the best-fit curve with the observational data set, as in Paper I.

\subsection{2. $H(z)$ and the relative galaxy ages}

In this section we discuss a possible observational determination of $H(z)$ based on the method developed by Jimenez et al. (2003), which involves differential age measurements. We present some constraints that can be placed on the evolution of our quintessence model by this data. First, it is worth pointing out some aspects connected with the sensitivity of the cosmology to the $t(z)$ and $\frac{\mathrm{d} z}{\mathrm{~d} t}$ relations. Actually, it is well known that in scalar tensor theories of gravity, as well as in general relativity, the expansion history of the universe is determined by the function $H(z)$. This implies that observational quantities, such as the luminosity distance, the angular diameter distance, and the lookback time, all depend on $H(z)$. It turns out that the most appropriate mathematical tool for studing the sensitivity of the cosmological model to such observables is the functional derivative of the corresponding relations with respect to the cosmological parameters (see Saini et al. 2003, for a discussion about this point in relation to distance measurements). However, from an empirical point of view, it is also possible to show that the lookback time

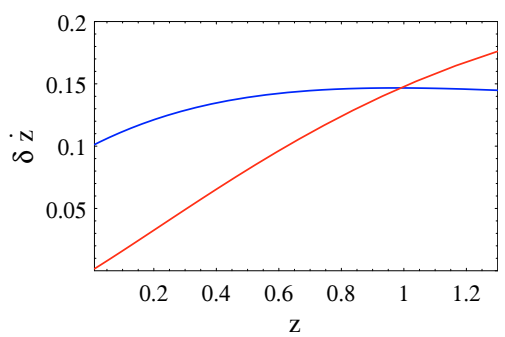

Fig. 15. The sensitivity of the $\frac{\mathrm{d} z}{\mathrm{~d} t}$ relation compared to the values of the parameters in our model. The red line shows $\delta \frac{\mathrm{d} z}{\mathrm{~d} t}=\frac{\frac{\mathrm{d} z}{\mathrm{~d} t}\left(s=-1.3, H_{0}=1\right)-\frac{\mathrm{d} z}{\mathrm{~d} t}\left(s=-1.4, H_{0}=1\right)}{\frac{\mathrm{d} z}{\mathrm{~d} t}\left(s=-1.4, H_{0}=1\right)}$, and the blue line shows $\delta \frac{\mathrm{d} z}{\mathrm{~d} t}=\frac{\frac{\mathrm{d} z}{\mathrm{~d} t}\left(s=-1.4, H_{0}=0.9\right)-\frac{\mathrm{d} z}{\mathrm{~d} t}\left(s=-1.4, H_{0}=1\right)}{\frac{\mathrm{d} z}{\mathrm{~d} t}\left(s=-1.4, H_{0}=1\right)}$.

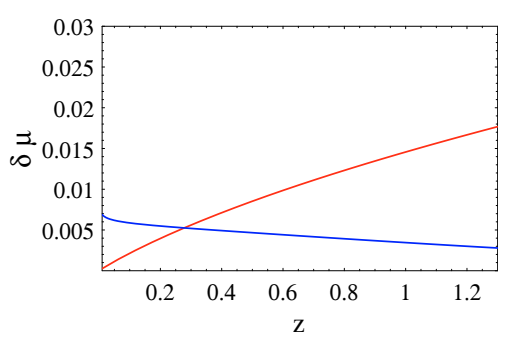

Fig. 16. The sensitivity of the $\mu=m-M$ relation compared to the values of the parameters in our model in. The red line shows $\delta \frac{\mathrm{d} \mu}{\mathrm{d} t}=\frac{\frac{\mathrm{d} \mu}{\mathrm{d} t}\left(s=-1.3, H_{0}=1\right)-\frac{\mathrm{d} \mu}{\mathrm{d} t}\left(s=-1.4, H_{0}=1\right)}{\frac{\mathrm{d} \mu}{\mathrm{d} t}\left(s=-1.4, H_{0}=1\right)}$, and the blue line shows $\delta \frac{\mathrm{d} \mu}{\mathrm{d} t}=\frac{\frac{\mathrm{d} \mu}{\mathrm{d} t}\left(s=-1.4, H_{0}=0.9\right)-\frac{\mathrm{d} \mu}{\mathrm{d} t}\left(s=-1.4, H_{0}=1\right)}{\frac{\mathrm{d} \mu}{\mathrm{d} t}\left(s=-1.4, H_{0}=1\right)}$.

is much more sensitive to the cosmological model than other observables, such as the luminosity distance and the distance modulus. This circumstance encourages us to use, together with the other more standard techniques discussed above, the age of cosmic clocks to test alternative cosmological scenarios. Apart from the advantage of providing an alternative investigation instrument, the age-based methods use the higher sensitivity to the cosmological parameters of the $\frac{\mathrm{d} z}{\mathrm{~d} t}$ relation, as shown in Figs. 15 and 16. Moreover, as we discuss in the following, such a method reveals its full strength when applied to old objects at very high $z$. Actually it turns out that this kind of analysis could remove, or at least reduce, the degeneracy that we observe at lower redshifts, for example the one in the Hubble diagram for SNIa observations, which can be fitted by different cosmological models with a similar statistical significance. Since the Hubble parameter can be related to the differential age of the universe as a function of redshift by the equation

$H(z)=-\frac{1}{1+z} \frac{\mathrm{d} z}{\mathrm{~d} t}$

a determination of $\mathrm{d} z / \mathrm{d} t$ directly measures $H(z)$. Jimenez et al. (2003) demonstrated the feasibility of this method by applying it to a $z \sim 0$ sample of galaxies. With the availability of new galaxy surveys, it becomes possible to determine $H(z)$ at $z>0$.

Here we use the $\mathrm{d} z / \mathrm{d} t$ data from (Simon et al. 2005) to determine $H(z)$ in the redshift range $0.1<z<1.8$. To follow the procedure described in Simon et al. (2005), first we group all galaxies together that are within $\Delta z=0.03$ of each other. This gives an estimate of the age of the universe at a given redshift. We then compute age differences only for those bins in redshift 


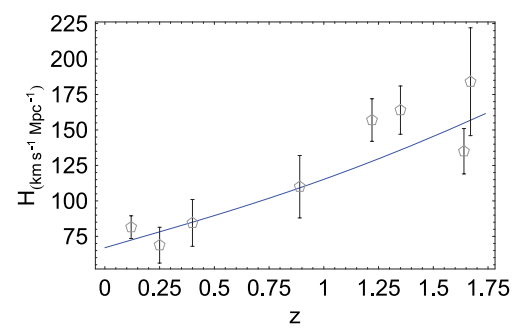

Fig. 17. The best-fit curve of the measured values of $H(z)$ corresponding to $\widehat{H}_{0}=1.01_{-0.03}^{+0.01}, s=-1.49_{-0.09}^{+0.03}$.

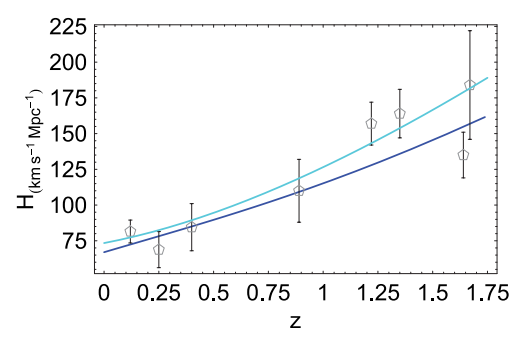

Fig. 18. The best-fit curve to the $H(z)$ data for our nmc model (dark blue line) and for the quintessence QCDM fitted to the new released WMAP- three years + SNLS data (WMAP New Three Year Results 2006) $\Omega_{\Lambda}=0.72 \pm 0.04, \Omega_{k}=-0.010_{-0.0009}^{+0.0016}, w=-1.06_{-0.08}^{+0.13}$ (blue line).

that are separated by more than $\Delta z=0.1$ but less than $\Delta z=0.15$. The first limit is imposed so that the age evolution between the two bins is larger than the error in the age determination. We note here that differential ages are less sensitive to systematic errors than absolute ages (see Jimenez et al. 2003). The observational value of $H(z)$ is then directly computed by using Eq. (77), and after that the ages data have been scaled according to our choice of the unit of time (the unknown scaling factor has been provided by the $\chi^{2}$ procedure). To determine the best-fit parameters, we define the following merit function:

$\chi^{2}\left(s, \widehat{H}_{0}\right)=\frac{1}{N-3} \sum_{i=1}^{N}\left[\frac{H\left(z_{i} ; s, \widehat{H}_{0}\right)-H_{i}}{\sigma_{i}}\right]^{2}$.

We obtain $\chi_{\text {red }}^{2}=1.09$ for 9 data points, and the best-fit values are $\widehat{H}_{0}=1.01_{-0.03}^{+0.01}, s=-1.49_{-0.09}^{+0.03}$. In Fig. 17 we compare the bestfit curve with the observational data set. It is interesting to note that future high redshift data could distinguish the degeneration among different models, since at high $z$ the predicted $H(z)$ more sensitively depends on the values of the parameters.

\subsection{The Sunyaev-Zeldovich/X-ray method}

In this section we discuss how the parameters of our model can be constrained by the angular diameter distance $D_{\mathrm{A}}$ as measured using the Sunyaev-Zeldovich effect (SZE) and the thermal bremsstrahlung (X-ray brightness data) for galaxy clusters. The distance measurements using Sunyaev-Zeldovich effect and $\mathrm{X}$-ray emission from the intracluster medium have to take into account that these processes depend on different combinations of some of the parameters of the clusters (see Birkinshaw 1999, and references therein). The SZE is a result of the inverse Compton scattering of the CMB photons on hot electrons of the intercluster gas, which preserves the number of photons but allows photons to gain energy thereby generating a decrement of the temperature in the Rayleigh-Jeans part of the black-body spectrum while an increment appears in the Wien region. We limit our analysis to the so-called thermal or static SZE. The kinematic effect, present only in clusters with a nonzero peculiar velocity with respect to the Hubble flow along the line of sight, will be neglected since the thermal SZE is typically an order of magnitude larger than the kinematic one. As in Paper I, we introduce the so-called Compton parameter, $y$, defined as the optical depth $\tau=\sigma_{\mathrm{T}} \int n_{\mathrm{e}} \mathrm{d} l$ times the energy gain per scattering:

$y=\int \frac{k_{B} T_{\mathrm{e}}}{m_{\mathrm{e}} c^{2}} n_{\mathrm{e}} \sigma_{\mathrm{T}} \mathrm{d} l$,

where $T_{\mathrm{e}}$ is the temperature of the electrons in the intracluster gas, $m_{\mathrm{e}}$ the electron mass, $n_{\mathrm{e}}$ the number density of the electrons, and $\sigma_{\mathrm{T}}$ is the Thompson cross section of electron scattering, and the integration is performed along the line of sight. In the lowfrequency regime of the Rayleigh-Jeans approximation, the shift of temperature is

$\frac{\Delta T_{\mathrm{RJ}}}{T_{0}} \simeq-2 y$,

where $T_{0}$ is the unperturbed CMB temperature. The next step in quantifing the SZE decrement is to specify the model for the intracluster electron density and temperature distribution, which appropriately describes the observational properties of the gas. Following Bonamente et al. (2005), we use a hydrostatic equilibrium double $\beta$-model. Actually, at the center of clusters the density may be high enough that the radiative cooling time-scale is less than the cluster's age, leading to a reduction in temperature and an increase in central density. This can increase the central X-ray emissivity. At large radii, the density of the gas is low enough that X-ray emission can be sustained for cosmological periods without significant cooling. Therefore, cool core clusters effectively exhibit two components: a centrally concentrated gas peak and a broad, shallower distribution of the gas. This phenomenon motivated the modeling of the gas density with a function of the form:

$n_{\mathrm{e}}(r)=n_{\mathrm{e} 0} \cdot\left[f\left(1+\frac{r^{2}}{r_{\mathrm{c} 1}^{2}}\right)^{-\frac{3 \beta}{2}}+(1-f)\left(1+\frac{r^{2}}{r_{\mathrm{c} 2}^{2}}\right)^{-\frac{3 \beta}{2}}\right]$.

The quantity $n_{\mathrm{e} 0}$ is the central density, $f$ governs the fractional contributions of the narrow and broad components $(0 \leq f \leq 1)$, $r_{\mathrm{c} 1}$ and $r_{\mathrm{c} 2}$ are the two core radii that describe the shape of the inner and outer portions of the density distribution, and $\beta$ determines the slope at large radii. The same $\beta$ is used for both the central and outer distribution in order to reduce the total number of degrees of freedom ${ }^{3}$. This shape generalizes the single $\beta$-model profile, introduced by Cavaliere \& Fusco-Femiano (1976) and commonly used to fit X-ray surface brightness profiles, to a double $\beta$-model of the density that has the freedom of following both the central spike in density and the gentler outer distribution. A double $\beta$-model of the surface brightness was first used by Mohr et al. (1999) to fit X-ray data of galaxy clusters, and the density model of Eq. (81) was further developed by La Roque et al. (2006). The X-ray surface brightness is related to the gas density as

$S_{\mathrm{X}}=\frac{1}{4 \pi(1+z)^{4}} \int n_{\mathrm{e}}^{2} \Lambda_{\mathrm{ee}} \mathrm{d} l$

3 It is worth noting that there are also different hydrodynamical models that predict universal gas density and gas temperature profiles that agree with the observations (see for instance the one illustrated in Komatsu \& Seljak (2001), derived from the universal dark matter density profile, assuming that the gas density traces the dark matter density in the outer parts of halos, or the one introduced in Rasia et al. (2004). 


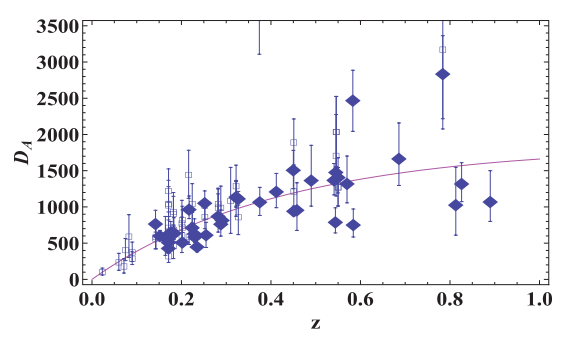

Fig. 19. Observational SZE data fitted to our model with the best-fit values $\widehat{H}_{0}=1_{-0.03}^{+0.01}, s=-1.49_{-0.09}^{+0.03}$, and $h=0.70 \pm 0.05$. The empty boxes indicate distance measurements for a sample of 44 mentioned clusters (see Birkinshaw 1999; Reese et al. 2002), while the filled diamonds indicate the measurement of the angular diameter distances from Chandra X-ray imaging and Sunyaev-Zel'dovich effect mapping of 39 high-redshift clusters (Bonamente et al. 2005).

where $z$ is the cluster redshift, $\Lambda_{\text {ee }}$ the X-ray cooling function, and it is a function of plasma temperature and energy in the rest frame of the cluster, including contributions from relativistic electron-ion thermal bremsstrahlung, electron-electron thermal bremsstrahlung, recombination, and two photon processes. The cluster angular diameter distance $D_{\mathrm{A}} \equiv \mathrm{d} l / \mathrm{d} \theta$, where $\theta$ is the line-of-sight angular size, can be inferred with a joint analysis of SZE, taking advantage of the different density dependence of the $\mathrm{X}$-ray emission and SZE decrement:

$$
\begin{gathered}
S_{\mathrm{X}} \propto \int n_{\mathrm{e}}^{2} \Lambda_{\mathrm{ee}} \mathrm{d} l=\int n_{\mathrm{e}}^{2} \Lambda_{\mathrm{ee}} D_{\mathrm{A}} \mathrm{d} \theta, \\
\Delta T_{\mathrm{CMB}} \propto \int n_{\mathrm{e}} T_{\mathrm{e}} \mathrm{d} l=\int n_{\mathrm{e}} T_{\mathrm{e}} D_{\mathrm{A}} \mathrm{d} \theta .
\end{gathered}
$$

It turns out that

$$
D_{\mathrm{A}} \propto \frac{\Delta T_{\mathrm{CMB}}^{2} \Lambda_{\mathrm{ee}}}{S_{\mathrm{X}} T_{\mathrm{e}}^{2}} .
$$

Note that $D_{\mathrm{A}}$ is proportional to $\Delta T_{\mathrm{CMB}}^{2}$ and $T_{\mathrm{e}}^{3 / 2}$ (since $\Lambda_{\mathrm{ee}} \propto$ $T_{\mathrm{e}}^{1 / 2}$ ), so the distance determination is strongly dependent on the accuracy of the SZE decrement and X-ray temperature measurements.

Recently distances to 18 clusters with redshift ranging from $z \sim 0.14$ to $z \sim 0.78$ have been determined from a likelihood joint analysis of SZE and X-ray observations (see Table 7 in Reese et al. 2002). Our analysis used angular diameter distance measurements for a sample of 83 clusters, containing the 18 above-mentioned clusters, another 24 known previously (see Birkinshaw 1999), and a recently released sample with the measurement of the angular diameter distances from the Chandra X-ray imaging and Sunyaev-Zel'dovich effect mapping of 39 high-redshift clusters of galaxies $(0.14 \leq z \leq 0.89)$ (Bonamente et al. 2005). The unprecedented spatial resolution of Chandra, combined with its simultaneous spectral resolution, allows a more accurate determination of distances. Let us consider the merit function of the form

$\chi^{2}\left(s, \widehat{H}_{0}\right)=\frac{1}{N-3} \sum_{i=1}^{N}\left[\frac{\left(D_{\mathrm{A}}\left(z_{i} ; s, \widehat{H}_{0}\right)-D_{i}\right)}{\sigma_{i}}\right]^{2}$.

Fitting the data we obtain $\chi_{\text {red }}^{2}=1.2$ for 83 data points, and the best-fit values are $\widehat{H}_{0}=1_{-0.03}^{+0.01}, s=-1.49_{-0.09}^{+0.03}$ and $h=$ $0.70 \pm 0.05$. In Fig. 19 we compare the best-fit curve with the observational SZE data.

\subsection{Gamma-ray burst Hubble diagram}

Gamma-ray bursts (GRBs) are bright explosions visible across most of the Universe, certainly out to redshifts of $z \sim 7$ and likely out to $z \sim 10$. Recent studies have pointed out that GRBs may be used as standard cosmological candles (Ghirlanda et al. 2004; Friedman \& Bloom 2005). It turns out that the energy released during bursts spans nearly three orders of magnitude, and the distribution of the opening angles of the emission, as deduced from the timing of the achromatic steepening of the afterglow emission, spans a similar wide range of values. However, when the apparently isotropic energy release and the conic opening of the emission are combined to infer the intrinsic, true energy release, the resulting distribution does not widen, as is expected for uncorrelated data, but shrinks to a very well-determined value (Frail \& Kulkarni 2003), with a remarkably small (one-sided) scattering, corresponding to about a factor of 2 in total energy. Similar studies in the X-ray band have reproduced the same results. It is thus very tempting to study to what extent this property of GRBs makes them suitable cosmological standard candles. Schaefer (2003) proposed to use the two well-known correlations of the GRBs luminosity (with variability and with time delay), while others have exploited the recently reported relationship between the beaming-corrected $\gamma$-ray energy and the locally observed peak energy of GRBs (see for instance Dai et al. 2004). As for the possible variation of ambient density from burst to burst, which may widen the distribution of bursts energies, Frail \& Kulkarni (2003) note that this spread is already contained in their data sample, and yet the distribution of energy released is still very narrow. There are at least two reasons GRBs are better than type Ia supernovae as cosmological candles. On the one hand, GRBs are easy to find and locate: even 1980s technology allowed BATSE to locate $\sim 1$ GRB per day, making the build-up of a 300-object database a one-year enterprise. The Swift satellite launched on 20 November 2004, detects GRBs at about the same rate as BATSE, but with a nearly perfect capacity for identifying their redshifts simultaneously with the afterglow observations ${ }^{4}$. Second, GRBs have been detected out to very high redshifts: even the current sample contains several events with $z>3$, with one (GRB 000131) at $z=4.5$ and another at $z=6.3$. This should be contrasted with the difficulty of locating $\mathrm{SN}$ at $z>1$ and absence of any SN with $z>2$. On the other hand, the distribution of luminosities of SNIa is narrower than the distribution of energy released by GRBs, corresponding to a magnitude dispersion $\sigma_{\mathrm{M}(\mathrm{SN})}=0.18$ rather than $\sigma_{\mathrm{M}(\mathrm{GRB})}=0.75$. Therefore GRBs may provide a complementary standard candle, out to distances that cannot be probed by SNIa, their major limitation being the larger intrinsic scatter of the energy released, as compared to the small scatter in peak luminosities of SNIa. There currently exists enough information to calibrate luminosity distances and independent redshifts for nine bursts (Schaefer 2003). These bursts were all detected by BATSE with redshifts measured from optical spectra of either the afterglow or the host galaxy. The highly unusual GRB980425 (associated with supernova SN1998bw) is not included because it is likely to be qualitatively different from the classical GRBs. Bursts with red shifts that were not recorded by BATSE still cannot have their observed parameters converted to energies and fluxes that are comparable with BATSE data. For the present analysis we use a sample of GRBs that had their redshifts estimated (Bloom et al. 2003), as represented in Fig. 20, with the distance modulus $\mu$, given by Eq. (72).

\footnotetext{
${ }^{4}$ http://swift.gsfc.nasa.gov
} 


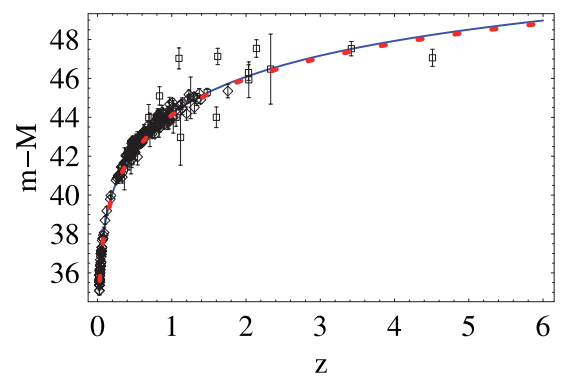

Fig. 20. Observational Hubble diagram for the recent SNIa sample compiled by Riess et al. (2006) (empty lozenges), and the GRBs data by Bloom et al. (2003) (empty boxes) fitted to our model. The solid curve is the best-fit curve with, $\widehat{H}_{0}=0.98_{-0.03}^{+0.03}, s=-1.43_{-0.04}^{+0.02}$. The red dashed line corresponds to the standard $\Lambda$ CDM model with $\Omega_{\Lambda}=0.71$.

To this aim, the only difference with respect to the SNIa is that we slightly modify the correction term of Eq. (73) and take

$\Delta m_{G_{\mathrm{eff}}}=2.5 \gamma \frac{\Delta G_{\mathrm{eff}}(t)}{(\ln 10) G_{\mathrm{eff}}}$.

We expect that $\gamma$ is of order unity, so that the $G$-correction would be roughly half a magnitude. We obtain $\chi_{\text {red }}^{2}=1.1$ for 24 data points and the best-fit value is $\widehat{H}_{0}=0.98_{-0.03}^{+0.03}, s=-1.43_{-0.04}^{+0.02}$, which are compatible with the SNIa results. We also find that $\gamma$, which appears in Eq. (86), is equal to 1.5. In Fig. 20 we compare the best-fit curve with both the GRBs and the SNIa Gold Sample.

\subsection{The gas fraction in clusters}

Measurements of the gas mass fraction in galaxy clusters have been proposed as a test of cosmological models (Allen et al. 2002). Both theoretical arguments and numerical simulations predict that the baryonic mass fraction in the largest relaxed galaxy clusters should not depend on the redshift and should provide an estimate of the cosmological baryonic density parameter $\Omega_{\mathrm{b}}$ (Eke et al. 1998). The baryonic content in galaxy clusters is dominated by the hot X-ray emitting intra-cluster gas so that what is actually measured is the gas mass fraction $f_{\text {gas }}$, and it is this quantity that should not depend on the redshift. Moreover, it is expected that the baryonic mass fraction in clusters is equal to the universal ratio $\Omega_{\mathrm{b}} / \Omega_{\mathrm{m}}$ so that $f_{\text {gas }}$ should indeed be given by $b \cdot\left(\Omega_{\mathrm{b}} / \Omega_{\mathrm{m}}\right)$, where the multiplicative factor $b$ is motivated by simulations that suggest that the gas fraction is lower than the universal ratio. Following the procedure described in Allen et al. (2002, 2004), and already used in Paper I we adopt the standard CDM model (i.e., a flat universe with $\Omega_{\mathrm{m}}=1$ and $h=0.5$ ) as a reference cosmology in making the measurements, so that the theoretical expectation for the apparent variation of $f_{\text {gas }}$ with the redshift is

$f_{\text {gas }}(z)=\frac{b \Omega_{b}}{(1+0.19 \sqrt{h}) \Omega_{\mathrm{m}}}\left[\frac{D_{\mathrm{A}}^{\mathrm{SCDM}}(z)}{D_{\mathrm{A}}^{\bmod }(z)}\right]^{1.5}$,

where we substitute the appropriate expression of $\Omega_{\mathrm{m}}$ for our model, and $D_{\mathrm{A}}^{\mathrm{SCDM}}$ and $D_{\mathrm{A}}^{\mathrm{mod}}$ are the angular diameter distance for the SCDM and our model respectively. Allen et al. (2002) have extensively analyzed the set of simulations in Eke et al. (1998) to get $b=0.824 \pm 0.089$, so we set $b=0.824$ in our analysis below. Actually, we checked that, for values in the $2 \sigma$ range

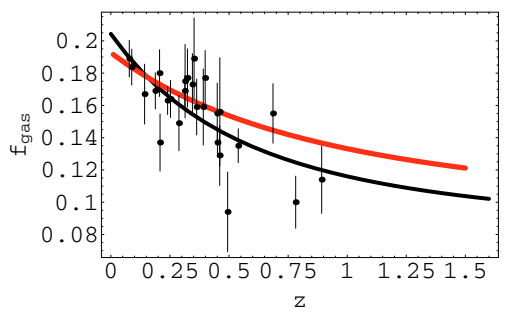

Fig. 21. The best-fit curve to the $f_{\text {gas }}$ data for our nmc model (red thick line) and for the quintessence model (black thick line) considered in Lima et al. (2003). It is interesting to note that, as pointed out also for the model described in Demianski et al. (2006), even if the statistical significance of the best-fit procedure for these two models is comparable, the best fit relative to our nmc model seems to be dominated by smaller redshift data, and the one relative to the Lima et al. model by higher redshift data.

quoted above, the main results do not depend on $b$. Moreover we defined the following merit function:

$\chi^{2}=\chi_{\text {gas }}^{2}+\left(\frac{\Omega_{\mathrm{b}} h^{2}-0.0214}{0.0020}\right)^{2}+\left(\frac{h-0.72}{0.08}\right)^{2}+\left(\frac{b-0.824}{0.089}\right)^{2}$,

where

$\chi_{\mathrm{gas}}^{2}=\sum_{i=1}^{N_{\mathrm{gas}}}\left[\frac{f_{\mathrm{gas}}\left(z_{i}, \alpha_{1}, \widehat{H}_{0}\right)-f_{\mathrm{gas}}^{\mathrm{obs}}\left(z_{i}\right)}{\sigma_{\mathrm{gi}}}\right]^{2}$.

Here $f_{\text {gas }}^{\mathrm{obs}}\left(z_{i}\right)$ is the measured gas fraction in galaxy clusters at redshift $z_{i}$ with an error $\sigma_{\text {gi }}$, and the sum is over the $N_{\text {gas }}$ clusters considered. Let us note that recently Allen et al. (2004) have released a catalog of 26 large relaxed clusters with a precise measurement of both the gas mass fraction $f_{\text {gas }}$ and the redshift $z$. We use these data to perform our likelihood analysis, we get $\chi^{2}=1.16$ for 26 data points, and $s=-1.39_{-0.01}^{+0.04}$, $\widehat{H}_{0}=0.98 \pm 0.04, h=0.65 \pm 0.05$, and $w_{\phi}=-0.95 \pm 0.07$. A brief comparison of our results with similar recent results from Lima et al. (2003), where the equation of state characterizing the dark energy component is constrained by using galaxy clusters $\mathrm{X}$-ray data and can still be done. Their analysis, however, considers quintessence models in the standard gravity theories, with a non evolving equation of state, but they allow the so-called phantom dark energy with $w<-1$, which violates the null-energy condition. As the best fit value of $w$ to the data of Allen et al. (2002) they obtain $w=-1.29_{-0.792}^{+0.686}$. In order to directly compare this result with our analysis, we first fit the model considered in Lima et al. (2003) to the updated and wider data set of Allen et al. (2004), used in our analysis. To this aim we also refer to the model function $f_{\text {gas }}(z)$ and the merit function $\chi^{2}$, defined in Eqs. (87) and (88), respectively. We get $\chi^{2}=1.175$ for 26 data points, and $\Omega_{\mathrm{m}}=0.23_{-0.03}^{+0.05}, h=0.76_{-0.09}^{+0.04}$, and $w=-1.11 \pm 0.35$, so $w<-1$, which corresponds to a phantom energy. Let us note that our model instead gives $w_{\phi}=-0.87 \pm 0.05$, what does not violate the null energy condition. In Fig. 21 we compare the best-fit curves for our and the Lima et al. model with the observational data.

\section{Growth of density perturbations}

In this section we consider the evolution of scalar density perturbations in the longitudinal gauge $\mathrm{d} s^{2}=-(1+2 \Phi) \mathrm{d} t^{2}+a^{2}(1-$ $2 \Phi) \mathrm{d} x^{2}$. It is well-known that in the framework of the minimallycoupled theory, where we have to deal with a fully relativistic 
component, that becomes homogeneous on smaller scales than the horizon, the standard quintessence cannot cluster on such scales. In the non minimally-coupled quintessence theories it is instead possible to separate a pure gravitational term both in the stress-energy tensor $T_{\mu \nu}$ and in the energy density $\rho_{\phi}$, so the situation changes, and it is necessary to consider also fluctuations of the scalar field. However, it turns out (Boisseau et al. 2000; Riazuelo \& Uzan 2002) that the equation for dust-like matter density perturbations inside the horizon can be written as follows

$\ddot{\delta}_{\mathrm{m}}+2 H \dot{\delta}_{\mathrm{m}}-\frac{1}{2} G_{\mathrm{Cav}} \rho_{\mathrm{m}} \delta_{\mathrm{m}} \simeq 0$,

where $G_{\mathrm{Cav}}$ is the effective gravitational constant defined by

$G_{\text {Cav }}=-\frac{1}{2 \xi(s) \phi^{2}}\left[\frac{1+16 \xi(s)}{1+12 \xi(s)}\right]$.

Equation (90) describes, in the non minimally coupled models, the evolution of the CDM density contrast, $\delta_{\mathrm{m}} \equiv \delta \rho_{\mathrm{m}} / \rho_{\mathrm{m}}$, for perturbations inside the horizon. In our model Eq. (90) is rather complicated and takes the form

$\ddot{\delta}_{\mathrm{m}}+2\left[\frac{t^{\frac{1}{s+3}-2-\frac{1}{s}}\left((s(2 s+9)+6) B(s) t^{\frac{3}{s+3}}+(2 s(s+3)+3)\right)}{A(s)}\right.$

$\left.\times\left(\left(\frac{1}{A(s)}\right)^{\frac{s}{s+1}}-B(s)\right)\left(\left(\frac{1}{A(s)}\right)^{\frac{s}{s+1}}+\left(t^{\frac{3}{s+3}}-1\right) B(s)\right)^{-1-\frac{1}{s}}\right] \dot{\delta}_{\mathrm{m}}$

$-\rho_{\mathrm{m}}\left[\frac{48(s+1)(s+2) t^{\frac{\left(s+\frac{3}{2}\right)^{2}}{4 s(s+3)}}}{2 C(s)^{2}(2 s+3)^{2}}\left(\frac{1+16 \xi(s)}{1+12 \xi(s)}\right)\right.$

$\left.\times\left(\left(\frac{1}{A(s)}\right)^{\frac{s}{s+1}}+B(s)\left(-\frac{12(s+1)(s+2) V t^{\frac{3}{s+3}}}{2 s+3}-1\right)\right)^{2+\frac{3}{s}}\right] \delta_{\mathrm{m}} \simeq 0$.

Equation (92) does not admit exact solutions and can only be solved numerically. However, since with our choice of normalization the whole history of the Universe is confined to the range $t \in[0,1]$; therefore, to study the behavior of solutions for $t \simeq 0$, we can always expand the functions in Eq. (92) in series around $t=0$, in order to get approximate solutions. Actually, we obtain an integrable Fuchsian differential equation, which is a hypergeometric equation. We then use such a solution to set the initial conditions at $t=0$ to numerically integrate Eq. (92) in the whole range $[0,1]$. We use the growing mode $\delta_{+}$and define the growth index $f$ as

$f \equiv \frac{\mathrm{d} \ln \delta_{+}}{\mathrm{d} \ln a}$,

where $a$ is the scale factor. Once we know how the growth in$\operatorname{dex} f$ evolves with redshift and how it depends on our model parameters, we can use the available observational data to estimate the values of these parameters and the present value of $\Omega_{\mathrm{m}}$. The 2dFGRS team has recently collected positions and redshifts of about 220000 galaxies and presented a detailed analysis of the two-point correlation function. They measured the redshift distortion parameter $\beta=\frac{f}{b}$, where $b$ is the bias parameter describing the difference in the distribution of galaxies and mass, and find that $\beta_{\mid z \rightarrow 0.15}=0.49 \pm 0.09$ and $b=1.04 \pm 0.11$. From the observationally determined $\beta$ and $b$, it is now straightforward to get the value of the growth index at $z=0.15$ corresponding to

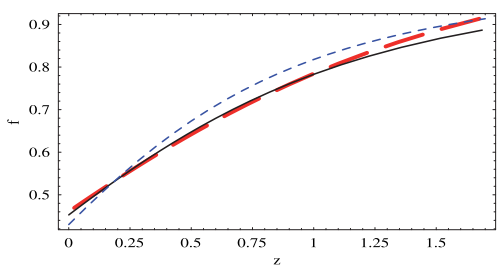

Fig. 22. The growth index $f$ in different cosmological models. The thick dashed red line corresponds to our non minimally-coupled model. The blue thin dashed curve corresponds to the standard $\Lambda$ CDM model with $\Omega_{\mathrm{m}}=0.25$, and the black solid line corresponds to another quintessence model with an exponential potential (described in Demianski et al. 2005).

the effective depth of the survey. Verde et al. (2001) used the bispectrum of 2dFGRS galaxies, and Lahav et al. (2002) combined the 2dFGRS data with CMB data, and they obtained

$b_{\text {verde }}=1.04 \pm 0.11$,

$b_{\text {lahav }}=1.19 \pm 0.09$.

Using these two values for $b$, we calculated the value of the growth index $f$ at $z=0.15$ to get, respectively,

$f_{1}=0.51 \pm 0.1$

$f_{2}=0.58 \pm 0.11$.

To evaluate the growth index at $z=0.15$ we first have to invert the $z-t$ relation and find $t(0.15)$ : actually the $z-t$ relation is rather involved and cannot be analytically inverted, so we perform this inversion numerically. Finally, we get $s=$ $-1.4 \pm 0.1, \widehat{H}_{0}=0.94_{-0.02}^{+0.05}, V_{0}=0.5 \pm 0.06$, which corresponds to $\Omega_{\Lambda_{\text {eff }}}=0.65 \pm 0.08$. In Fig. 22 we show how the growth index is changing with redshift in our non minimally-coupled model as compared with the standard $\Lambda$ CDM model, with $\Omega_{\mathrm{m}}=0.25$, and a quintessence model, the minimally-coupled exponential model described in Demianski et al. (2005). We note that at low redshift, theoretical predictions of these different models are not distinguishable, so independent measurements from large redshift surveys at different depths could disentangle this degeneracy.

\section{Summary and conclusions}

In this paper we have extended the analysis that we performed in Paper I (where we have analyzed a special extended quintessence model, based on one of the most commonly used quintessence potentials $V(\phi)=\lambda \phi^{4}$, corresponding to the coupling $\left.F(\phi)=(3 / 32) \phi^{2}\right)$, considering a new and wider class of theories for which exact solutions of the Einstein equations are known. We also discussed how it is possible in such models to treat the fine-tuning problem in an alternative way. We have shown that an epoch of accelerated expansion appears in a natural way in the family of such models selected by requiring that their corresponding point like Lagrangian admits a Noether symmetry. In the non minimally-coupled scalar tensor theory of gravity, it is possible to perform an appropriate conformal transformation and to move from the Jordan picture to the standard Einstein one, but then matter becomes coupled to the scalar field. We explored both descriptions and also considered the neutrino mass varying model as a possible example of non minimallycoupled scalar tensor theory.

It turns out that the imposed requirement of a Noether symmetry is quite restrictive, so we obtained a family of models that is fully specified by 3 parameters: a parameter $s$ that determines 
Table 2. The basic cosmological parameters derived from our model are compared with observational data.

\begin{tabular}{ccccc}
\hline \hline Dataset & $s$ & $\widehat{H}_{0}$ & $\Omega_{\Lambda_{\mathrm{eff}}}$ & $w_{\phi}$ \\
\hline high redshift SNIa & $-1.46_{-0.03}^{+0.04}$ & $0.96_{-0.05}^{+0.05}$ & $0.69_{-0.06}^{+0.04}$ & $-1.01 \pm 0.02$ \\
dimensionless coordinates test & $-1.49_{-0.04}^{+0.02}$ & $0.97_{-0.03}^{+0.04}$ & $0.73 \pm 0.08$ & $-0.98 \pm 0.03$ \\
relative galaxy ages & $-1.49_{-0.09}^{+0.03}$ & $1.01_{-0.03}^{+0.01}$ & $0.80 \pm 0.07$ & $-0.99 \pm 0.04$ \\
sze data & $-1.49_{-0.05}^{+0.03}$ & $1_{-0.03}^{+0.01}$ & $0.70 \pm 0.05$ & $-1 \pm 0.04$ \\
GRBs dataset & $-1.43_{-0.04}^{+0.02}$ & $0.98_{-0.03}^{+0.03}$ & $0.76 \pm 0.06$ & $-0.9 \pm 0.04$ \\
fraction of gas in clusters & $-1.39_{-0.01}^{+0.04}$ & $0.98 \pm 0.04$ & $0.77_{-0.03}^{+0.05}$ & $-0.87 \pm 0.05$ \\
galaxies peculiar velocity & $-1.4 \pm 0.1$ & $0.94_{-0.02}^{+0.05}$ & $0.65 \pm 0.08$ & $-0.85 \pm 0.04$ \\
average & $-1.46 \pm 0.09$ & $0.97 \pm 0.02$ & $0.74 \pm 0.03$ & $-1.01 \pm 0.02$ \\
\hline
\end{tabular}

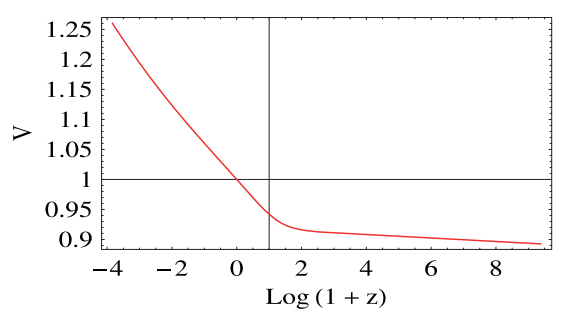

Fig. 23. The potential $V$ as a function of the redshift $z$.

the strength of the non-minimal coupling and the potential of the scalar field, $H_{0}$ as the Hubble constant, and a parameter $V_{0}$ that determines the scale of the potential. To determine the values of these parameters, we compared predictions of our model with several independent observational data. The results of this parameter determination procedure are presented in Table 2 . We see that with our average value of $s=-1.46$ the scale factor, for small $t$, is changing as $a \sim t^{0.66} \sim t^{2 / 3}$, and for large $t$, as $a \sim t^{1.25} \sim t^{5 / 4}$ while $\phi \sim t^{0.0032}$ and $\phi \sim t^{0.083}$ in corresponding asymptotic regimes. The potential $V$ decays to zero for large $t$, after reaching a maximum value (see Fig. 23). Similarly, the effective gravitational coupling $G_{\text {eff }}=-\frac{1}{2 F}$ decreases for large $t$, until it becomes zero for $t \rightarrow \infty$ (we have a sort of asymptotic freedom at $t \rightarrow \infty$ ). It turns out that in our model the observational constraints on the variation of the effective gravitational constant are respected. Actually a new analysis of the Big Bang Nucleosynthesis (Copi et al. 2004) restricts the variations in $G$ to

$\Delta G_{\mathrm{BBN}} / G_{0}=0.01_{-0.16}^{+0.20}$

at a $68 \%$ confidence level, where $\Delta G_{\mathrm{BBN}} / G_{0}=\frac{G_{\mathrm{BBN}}-G_{0}}{G_{0}}$, and $G_{0} \equiv G_{\text {Newton}}$. The combined analysis of the new ${ }^{4} \mathrm{He}$ and WMAP data implies that

$-0.10<\Delta G_{\mathrm{BBN}} / G_{0}<0.13$.

A recent analysis of the secular variation of the period of nonradial pulsations of the white dwarf G117-B15A shows (Benvenuto et al. 2004) that $0<\dot{G} / G<4.0 \times 10^{-11} \mathrm{yr}^{-1}$ at $2 \sigma$, which is of the same order of magnitude as previous independent bounds (see also Biesiada \& Malec 2004). With our unit of time this becomes $0<\dot{G} / G<4.0 \tau \times 10^{-2}$, where $\tau=13.73_{-0.15}^{+0.16}$ as given by the WMAP team (Spergel et al. 2006).

Initially, for small $t$, the matter energy density is higher than the energy density of the scalar field. In Table 2 we present results of our analysis, and they show that predictions of our model are fully compatible with the recent observational data. Comparing results of this paper with our previous analysis of minimally coupled scalar field models (see Paper I), we conclude that the present-day observational data connected with the post recombination evolution of the universe can be fitted by several different models of quintessence. More data on high-redshift supernovae of type Ia and GRBs are needed, as is more information on the early phase of structure formation in order to place stronger restrictions on the allowed type of dark energy.

Acknowledgements. This work was supported in part by the grant of Polish Ministry of Science and Higher Education 1-P03D-014-26, and by INFN Na12. The authors are very grateful to Professor Djorgovski for providing the data that we used in Sect. 3.1.1, and to Professors Verde and Simon, for providing the data used in Sect. 3.2.2. Of course we take full responsibility for the fitting procedure.

\section{References}

Accetta, F. S., Zoller, D. J., \& Turner, M. S. 1985, Phys. Rev. D, 31, 3064

Acquaviva, V., Baccigalupi, C., Leach, S. M., Liddle, A. R., \& Perrotta, F. 2005, Phys. Rev. D, 71, 104025

Allen, S. W., Schmidt, R. W., \& Fabian, A. C. 2002, MNRAS, 334, L11

Allen, S. W., Schmidt, R. W., Ebelling, H., Fabian, A. C., \& van Speybroeck, L. 2004, MNRAS, 353, 457

Amendola, L. 1999, Phys.Rev. D, 60, 043501

Amendola, L., Gasperini, M., \& Piazza, F. 2004, JCAP, 9, 014

Astier, P., Guy, J., Regnault, N., et al. 2006, A\&A, 447, 31

Benvenuto, O. G., Garcia-Berro, E., \& Isern, J. 2004, Phys. Rev. D, 69, 082002

Bertotti, B., Iess, L., \& Tortora, P. 2003, Nature, 425, 374

Bi, X.-J., Gu, P., Wang, X., \& Zhang, X. 2004, Phys. Rev. D, 69, 113007

Biesiada, M., \& Malec, B. 2004, MNRAS, 350, 644

Birkinshaw, M. 1999, Phys. Rep., 310, 97

Bloom, J. S., Frail, D. A., \& Kulkarni, S. R. 2003, ApJ, 594, 674

Bonamente, M., Joy, M., La Roque, S., et al. 2005 [arXiv: astro-ph/0512349]

Brookfield, A. W., van de Bruck, C., Mota, D. F., \& Tocchini-Valentini, D. 2006a, Phys. Rev. Lett., 96, 061301

Brookfield, A. W., van de Bruck, C., Mota, D. F., \& Tocchini-Valentini, D. 2006b, Phys. Rev. D, 73, 083515

Boisseau, B., Esposito-Farese, G., Polarski, D., \& Starobinsky, A. A. 2000, Phys. Rev. Lett., 85, 2236

Caldwell, R. R., Dave, R., \& Steinhardt, P. J. 1998, Phys. Rev. Lett., 80, 1582

Caldwell, R. R., Kamionkowski, M., \& Weinberg, N. N. 2003, Phys. Rev. Lett., 91, 071301

Capozziello, S., de Ritis, R., Rubano, C., \& Scudellaro, P. 1996, Riv. Nuovo Cimento, 19, 4

Capozziello, S., de Ritis, R., \& Marino, A. A. 1997, Class. Quant. Grav., 14, 3243

Cavaliere, A., \& Fusco-Femiano, R. 1976, 49, 137

Cavaliere, A., \& Fusco-Femiano, R. 1978, A\&A, 70, 677

Clifton, T., Mota, D. F., \& Barrow, J. D. 2005, MNRAS, 358, 601

Copi, C. J., Davis, A. N., \& Krauss, L. M. 2004, Phys. Rev. Lett., 92, 171301

Crooks, J. L., \& Frampton, P. H. 2006, Phys. Rev. D, 73, 123512

Cyburt, R. H., Fields, B. D., Olive, K. A., \& Skillman, E. 2005, Astropart. Phys., 23, 313

Dai, Z. G., Liang, E. W., \& Xu, D. 2004, ApJ, 612 L101

Daly, R. A., \& Djorgovski, S. G. 2004, ApJ, 612, 652

Daly, R., \& Djorgovski, S. G., 2005 [arXiv:astro-ph/0512576]

Das, S., Corasaniti, P., \& Khoury, J. 2006, Phys. Rev. D, 73, 083509

De La Macorra, A., Melchiorri, A., Serra, P., \& Bean, R. 2007, Astroparticle Physics, 27, 406

Demianski, M., de Ritis, R., Marmo, G., Platania, G., \& Stornaoiolo, C. 1991, Phys. Rev. D, 44, 3136

Demianski, M., de Ritis, R., Marino, A. A., \& Piedipalumbo, E. 2003, A\&A, 411, 33 
Demianski, M., Piedipalumbo, E., Rubano, C., \& Tortora, C. 2005, A\&A, 431, 27

Demianski, M., Piedipalumbo, E., Rubano, C., \& Tortora, C. 2006, A\&A, 454, 55

de Ritis, R., Marino, A. A., Rubano, C., \& Scudellaro, P. 2000, Phys. Rev. D, 62, 043506

Damour, T., \& Esposito-Farese, G. 1993, Phys. Rev. Lett., 70, 2220

Eke, V., Navarro, J. F., \& Frenk, C. S. 1998, ApJ, 503, 569

Elliott, S. R., \& Engel, J. 2004, J.Phys. G, 30, R 183

Ellis, J., Lahanas, A. B., Nanopoulos, D. V., \& Tamvakis, K. 1984, Phys. Lett. B, 134, 429

Esposito Farese, G. 2004, AIP Conf. Proc., 736, 35

Faraoni, V. 2000, Phys. Rev. D, 62, 023504

Fardon, R., Nelson, A. E., \& Weiner, N. 2004, JCAP 0410:005

Frail, D. A., \& Kulkarni, S. R. 2003, ApJ, 594, 674

Friedman, A. S., \& Bloom J. S. 2005, ApJ, 627, 1

Garcia-Bellido, J. 1997, Phys. Rev. D, 55, 4603

Gaztañaga, E., Garcia-Berro, E., Isern, J., Bravo, E., \& Dominguez 2001, Phys. Rev. D, 1

Ghirlanda, G., Ghisellini, G., \& Lazzati, D. 2004, ApJ, 616, 331

Hannestad, S. 2005, Phys. Rev. Lett., 95, 221301

Hu, W. 2005, Phys. Rev. D, 71, 047301

Jimenez, R., Verde, L., Treu, T., \& Stern, D. 2003, ApJ, 593, 622

Kaplan, D. B., Nelson, A. E., \& Weiner, N. 2004, Phys. Rev. Lett., 93, 091801

Klapdor-Kleingrothaus, H. V. 2006, talk at SNOW, 2nd Scandinavian Neutrino Workshop (Stockholm, Sweden)

Klapdor-Kleingrothaus, H. V., Krivosheina, I. V., Dietz, A., \& Chkvorets, O. 2004, Phys. Lett. B, 586, 198

Komatsu, E., \& Seljak, U. 2001, MNRAS, 327, 1353

Lahav, O., Bridle, S. L., Percival, W. J., \& the 2dFGRS Team 2002, MNRAS, 333, 961

La Roque, S., Bonamente, M., Joy, M., et al. 2006, ApJ, 652, 917

Lesgourgues, J., \& Pastor, S. 2006, Phys. Rep., 429, 307

Lima, J. A. S., Cunha, J. V., \& Alcaniz, J. S. 2003, Phys. Rev., D, 68, 023510

Marino, A. A., \& de Ritis, R. 2001, Phys. Rev. D, 64, 083509

Masiero, A., Pietroni, M., \& Rosati, P. 2000, Phys. Rev. D, 61, 023504

Mohr, J., Mathiesen, B., \& Evrard, A. 1999, ApJ, 517, 627

Peebles, P. J. E., \& Ratra, B. 1988, Astrophys. J. Lett., 325, L17

Perlmutter, S., Gabi, S., Goldhaber, G., et al. 1997, ApJ, 483, 565

Rasia, E., Tormen, G., \& Moscardini, L. 2004, MNRAS, 351, 237

Reese, E., Carlstrom, J. E., Joy, M., et al. 2002, ApJ, 581, 53

Riazuelo, A., \& Uzan, J.-P. 2002, Phys. Rev. D, 66, 023525
Riess, A. G. 2000, PASP, 112, 1284

Riess, A. G., Filippenko, A. V., Challis, P., et al. 1998, AJ, 116, 1009

Riess, A. G., Strolger, L.-G., Tonry, J., et al. 2004, ApJ, 607, 665

Riess, A. G., Strolger, L.-G., Casertano, S., et al. 2007

[arXiv: astro-ph/0611572]

Rubano, C., \& Scudellaro, P. 2002, Gen. Rel. Grav., 34, 307

Rubano, C., Scudellaro, P., Piedipalumbo, E., Capozziello, S., \& Capone, M. 2004, Phys. Rev. D, 69, 103510

Saini, T. D., Padmanabhan, T., \& Bridle, S. 2003, MNRAS, 343, 533

Sanyal, A. K., \& Modak, B. 2001, Class. Q. Grav. 18, 3767

Sanyal, A. K., Rubano, C., \& Piedipalumbo, E. 2003, Gen. Rel. Grav., 35, 1617

Sarazin, C. L. 1988, X-Ray Emission from Cluster of Galaxies (Cambridge: Cambridge Univ. Press)

Schaefer, B. E. 2003, ApJ, 583, L67

Schimd, C., Uzan, J. P., \& Riazuelo, A. 2005, Phys. Rev. D, 71, 083512

Schmidt, B. P., Suntzeff, N. B., Phillips, M. M., et al. 1998, ApJ, 507, 46

Shapiro, I. I. 1993, in General Relativity and Gravitation 12, ed. N. Ashby, et al., Cambridge University Press

Shapiro, S. S., Davis, J. L., Lebach, D. E., \& Gregory, J. S. 2004, Phys. Rev. Lett., 92, 121101

Shaw, D. J., \& Barrow, J. D. 2005, Phys. Lett. B, 639, 596

Simon, J., Jimenez, R., \& Verde, L. 2005, Phys. Rev. D, 71, 123001

Spergel, D. N., Bean, R., Dorè, O., et al. 2006, ApJ, sumitted

Steinhardt, P. J., Wang, L., \& Zlatev, I. 1999, Phys. Rev. D, 59, 123504

Tonry, J. L., Schmidt, B. P., Barris, B., et al. 2003, ApJ, 594, 1

Torres, D. F. 2002, Phys. Rev. D, 66, 043522

Urena-Lopez, L. A., \& Matos, T. 2000, Phys. Rev. D, 62, 081302

Uzan, J. P. 1999, Phys. Rev. D, 59, 123510

Uzan, J. Ph. 2003, Rev. Mod. Phys., 75, 403

Verde, L., Kamionkowski, M., Mohr, J. J., \& Benson, A. J. 2001, MNRAS, 321, L7

Vikman, A. 2005, Phys. Rev. D, 71, 023515

Wang, L., Caldwell, R. R., Ostriker, J. P., \& Steinhardt, P. J. 2000, ApJ, 530, 17

Weinberg, S. 1989, Rev. Mod. Phys., 61, 1

Will, C. M. 1993, Theory and Experiments in Gravitational Physics (Cambridge: Cambridge Univ. Press)

Williams, J. G., Newhall, X. X., Dickey, J. O., et al. 1996, Phys. Rev. D, 53, 6730

WMAP data at http: //lambda.gsfc.nasa.gov

Zee, F. 1979, Phys. Rev. Lett., 42, 417

Zeldovich, Ya. B. 1967, Pis'ma Zh. Eksp. Teor. Fiz., 6, 883 (1967, JETP Lett. 6, 316) 